\title{
UV-TO-FIR ANALYSIS OF SPITZER/IRAC SOURCES IN THE EXTENDED GROTH STRIP. I. MULTI-WAVELENGTH PHOTOMETRY AND SPECTRAL ENERGY DISTRIBUTIONS
}

\author{
Guillermo Barro ${ }^{1}$, P. G. Pérez-González ${ }^{1,5}$, J. Gallego ${ }^{1}$, M. L. N. Ashby ${ }^{2}$, M. Kajisawa ${ }^{3}$, S. Miyazaki ${ }^{4}$, V. Villar ${ }^{1}$, \\ T. YAMADA ${ }^{3}$, AND J. ZAMORANO ${ }^{1}$ \\ ${ }^{1}$ Departamento de Astrofísica, Facultad de CC. Físicas, Universidad Complutense de Madrid, E-28040 Madrid, Spain \\ ${ }^{2}$ Harvard-Smithsonian Center for Astrophysics, 60 Garden Street, Cambridge, MA 02138, USA \\ ${ }^{3}$ Astronomical Institute, Tohoku University, Aramaki, Aoba, Sendai 9808578, Japan \\ ${ }^{4}$ National Astronomical Observatory of Japan, Mitaka, Tokyo 181-8588, Japan \\ Received 2010 May 21; accepted 2011 January 14; published 2011 March 1
}

\begin{abstract}
We present an IRAC 3.6+4.5 $\mu$ m selected catalog in the Extended Groth Strip (EGS) containing photometry from the ultraviolet to the far-infrared and stellar parameters derived from the analysis of the multi-wavelength data. In this paper, we describe the method used to build coherent spectral energy distributions (SEDs) for all the sources. In a forthcoming companion paper, we analyze those SEDs to obtain robust estimations of stellar parameters such as photometric redshifts, stellar masses, and star formation rates. The catalog comprises 76,936 sources with [3.6] $\leqslant 23.75 \mathrm{mag}$ ( $85 \%$ completeness level of the IRAC survey in the EGS) over $0.48 \mathrm{deg}^{2}$. For approximately $16 \%$ of this sample, we are able to deconvolve the IRAC data to obtain robust fluxes for the multiple counterparts found in ground-based optical images. Typically, the SEDs of the IRAC sources in our catalog count with more than 15 photometric data points, spanning from the ultraviolet wavelengths probed by GALEX to the far-infrared observed by Spitzer, and going through ground- and space-based optical and near-infrared data taken with 2-8 $\mathrm{m}$ class telescopes. Approximately $95 \%$ and $90 \%$ of all IRAC sources are detected in the deepest optical and near-infrared bands. These fractions are reduced to $85 \%$ and $70 \%$ for $\mathrm{S} / \mathrm{N}>5$ detections in each band. Only $10 \%$ of the sources in the catalog have optical spectroscopy and redshift estimations. Almost 20\% and $2 \%$ of the sources are detected by MIPS at 24 and $70 \mu \mathrm{m}$, respectively. We also cross-correlate our catalog with public X-ray and radio catalogs. Finally, we present the Rainbow Navigator public Web interface utility, designed to browse all the data products resulting from this work, including images, spectra, photometry, and stellar parameters.
\end{abstract}

Key words: galaxies: high-redshift - galaxies: photometry - galaxies: starburst - infrared: galaxies

Online-only material: machine-readable tables

\section{INTRODUCTION}

Multi-wavelength observations of blank fields provide a fertile ground for studies of the evolution of galaxies from the early universe. Indeed, in the past decade we have advanced amazingly in our knowledge about the formation of galaxies thanks to deep field imaging and spectroscopic surveys. The extraordinary success of these surveys is sustained by the coordinated effort of several telescope facilities, institutions, and research groups that gather large collections of multiwavelength photometry and spectroscopy, providing the entire scientific community with a vast pool of data to analyze. Remarkable examples of this kind of projects are the Hubble Deep Field (HDF) observations (Williams et al. 1996), the Classifying Objects by Medium-Band Observations project (COMBO17; Wolf et al. 2001), Great Observatories Optical Deep Survey (GOODS; Giavalisco et al. 2004), VIMOS-VLT Deep Survey (VVDS; Le Fèvre et al. 2005), All-wavelength Extended Groth strip International Survey (AEGIS; Davis et al. 2007), or Cosmic Evolution Survey (COSMOS; Scoville et al. 2007).

Nevertheless, the full scientific exploitation of these surveys unavoidably needs a consistent merging of the data coming from heterogeneous sources (with different depths and resolutions) to build catalogs of galaxies characterized with panchromatic photometry and spectroscopy. Although substantial effort has been

\footnotetext{
5 Associate Astronomer at Steward Observatory, The University of Arizona.
}

devoted to create homogeneously processed multi-wavelength catalogs for the most important fields (e.g., Chandra Deep Field South, Wolf et al. 2001; Hubble Deep Field North, Steidel et al. 2003, Capak et al. 2004; Extended Groth Strip (EGS), Coil et al. 2004, Ilbert et al. 2006), many of these catalogs are selected in the optical bands (i.e., rest-frame ultraviolet (UV) at high redshift) and lack near-infrared (NIR) imaging (an important data set for the studies of galaxy populations at high redshift) reaching depths that match the optical observations. Fortunately, the proliferation of deep and wide NIR surveys has supported the publication of an increasing number of multi-band samples selected in the $K$ band (Grazian et al. 2006; Quadri et al. 2007; Wuyts et al. 2008) or Infrared Array Camera (IRAC) bands (Rowan-Robinson et al. 2008; Pérez-González et al. 2005, 2008, hereafter PG05 and PG08). A detailed UV-to-NIR coverage of the spectral energy distribution (SED) improves the estimates of important stellar parameters, such as the mass, age, extinction, or star formation rate (SFR; see, e.g., Walcher et al. 2008; Salim et al. 2007). Moreover, in order to obtain the most reliable stellar mass estimates for $z \sim 2-3$ galaxies, and to distinguish young dusty starburst from quiescent galaxies at these redshifts, we need to obtain data probing the rest-frame NIR for these populations (Bundy et al. 2006; Pozzetti et al. 2007; Wuyts et al. 2007; Williams et al. 2009; Muzzin et al. 2009). This became possible with the launch of Spitzer and the use of one of its instruments, IRAC (Fazio et al. 2004), which covers the 3.6-to- $8.0 \mu \mathrm{m}$ spectral range. 
The power of multi-band catalogs is significantly enhanced when optical and NIR data are complemented with mid-IR $(>5 \mu \mathrm{m})$ to radio fluxes, such as those from Spitzer/MIPS, SCUBA, VLA, or Herschel surveys. These data directly probe the emission of the dust component of galaxies (Santini et al. 2009; Wuyts et al. 2008). Actively star-forming galaxies harbor large amounts of dust, which cause that a fraction of the UV emission, directly related to the ongoing star formation, is extincted and re-emitted in the IR. Thus, modeling the IR emission offers not only a complementary approach to estimate the SFR of a galaxy but also an improved measurement of the intrinsic UV extinction (Reddy et al. 2006; Daddi et al. 2007; Salim et al. 2007; Iglesias-Páramo et al. 2007). The IR approach to studies of the star formation becomes particularly relevant at higher redshifts, where the number of luminous infrared galaxies (whose integrated IR luminosity is $L(\mathrm{IR})>10^{11} L_{\odot}$ ) and their contribution to the cosmic SFR density increase significantly (Chary \& Elbaz 2001; PG05; Caputi et al. 2007).

The downside to having an exceptional data pool available in many regions of the sky is that the data quality is largely heterogeneous. Unfortunately, the high-redshift community still lacks the existence of a unified database that facilitates the access to the multiple data sets and resources, similar to local extragalactic databases such as the NASA Extragalactic Database (NED) or the Sloan Digitalized Sky Survey (SDSS; York et al. 2000) database.

The purpose of this work is to present an NIR-selected sample of galaxies with well-sampled SEDs and analyze their properties maximally benefiting from the panchromatic data. To do this, we have built an IRAC 3.6+4.5 $\mu \mathrm{m}$ selected photometric and spectroscopic catalog including data from X-ray to radio wavelengths for 76,936 galaxies at $0<z \lesssim 4$ in the EGS.

The EGS has been intensively observed as a part of the AEGIS (Davis et al. 2007) collaboration in order to assemble an exceptional multi-wavelength data set, including deep optical imaging from the Canada-France-Hawaii Telescope (CFHT) Legacy Survey (CFHTLS), HST coverage in two bands, UV data from Galaxy Evolution Explorer (GALEX), and mid-IR and far-IR photometry from Spitzer. In addition, the EGS is the key field for the DEEP2 survey, one of the largest and deepest spectroscopic surveys to date (Davis et al. 2003), with more than 10,000 optical spectra down to $R \sim 24$. This vast data set converts the EGS in one of the main fields for the study of galaxy evolution at different epochs of the lifetime of the universe. Our goal is get advantage of this impressive multi-wavelength data collection and use it to build UV-to-FIR SEDs, whose analysis will allow us to obtain estimations of interesting parameters, such as the photometric redshifts, stellar masses, and SFRs.

The photometric catalog along with the photometric redshifts and the inferred stellar parameters are intended to become a multi-purpose resource useful for many different scientific goals. Some of them will be presented in forthcoming papers. We make all the catalogs publicly available through our Web site and through a dedicated Web interface, dubbed Rainbow Navigator, conceived to facilitate the access to the data, but also to serve as a permanent repository for updates in these catalogs, or similar catalogs in other cosmological fields (e.g., those presented in PG08 for the GOODS fields).

In this paper, we concentrate on the description of the data set and the methods developed to measure the merged photometry for the IRAC sample in the EGS. We also analyze the multi-band properties of the sample to understand the main properties of the Spitzer surveys. All the multi-wavelength photometry is released in a public database, conceived to allow the astronomical community to access all the results from our work and use them for their own purposes. In a forthcoming companion paper (hereafter Paper II), we will present our methodology to fit the SEDs presented in this paper and to estimate photometric redshifts, stellar masses, and SFRs out of them. We will also assess the quality of the inferred parameters, analyzing in detail their intrinsic systematic and random uncertainties.

The outline of this paper follows. In Section 2, we present the available data sets that we have compiled for this paper. In Section 3, we present the techniques developed to extract the IRAC $3.6+4.5 \mu \mathrm{m}$ selected sample and we discuss the properties of the IRAC photometry of the sample. In Section 4, we present the methods developed to build the merged multiband photometric catalog. In Section 5, we describe in detail the photometric properties and reliability of the catalog. In Section 6, we describe the format of the published catalogs, the database built to allow an easy access and handling of those, baptized as the Rainbow Cosmological Surveys Database, and the publicly available Web interface to surf the database, Rainbow Navigator.

Throughout this paper we use $\mathrm{AB}$ magnitudes. We adopt the cosmology $H_{0}=70 \mathrm{~km}^{-1} \mathrm{~s}^{-1} \mathrm{Mpc}^{-1}, \Omega_{m}=0.3$, and $\Omega_{\lambda}=0.7$.

\section{DATA DESCRIPTION}

The EGS $\left(\alpha=14^{\mathrm{h}} 17^{\mathrm{m}}, \delta=+52^{\circ} 30^{\prime}\right)$ is one of the most targeted cosmological deep fields. Noticeably, a comprehensive panchromatic data set has been compiled in this field within the AEGIS collaboration (Davis et al. 2007).

The sample of galaxies studied in this paper is based on an IRAC $3.6+4.5 \mu \mathrm{m}$ selection. This choice obeys to several reasons. First, the IRAC bands are specially tailored to probe the rest-frame near-infrared (NIR) fluxes of distant galaxies, thus being the perfect tool for studies of massive galaxies at high- $z$ (e.g., Rodighiero et al. 2007; Mancini et al. 2009). Second, the quality, depth, and ubiquity of the IRAC observations in the socalled cosmological fields favor the assembly of coherent flux limited catalogs over large cosmological volumes (e.g., PG08), which is the cornerstone of the observational cosmology. The IRAC bands offer an alternative to the less efficient NIR groundbased surveys and provide a starting point to consistently anchor surveys at longer wavelengths (Spitzer/MIPS, Herschel, ALMA). Despite its lower spatial resolution (FWHM $\sim 2^{\prime \prime}$; Fazio et al. 2004) compared to optical/NIR ground-based surveys, the image quality of IRAC is very stable and several authors have been able to perform deblending techniques successfully merging IRAC catalogs into their panchromatic data sets (PG05; PG08; Grazian et al. 2006; Ilbert et al. 2009).

In the rest of this section, we describe the multi-wavelength data sets in EGS compiled for this paper to characterize the IRAC-selected sample. Table 1 summarizes the main characteristics of these data sets, including the depth, the area, and the image quality. Figure 1 shows the footprints of the various surveys, highlighting the area with higher band coverage.

\subsection{X-ray Data}

The EGS region has been observed in the X-ray $(0.5-10 \mathrm{keV})$ using Chandra/ACIS during two observation cycles in 2002 and 2005. The AEGIS-X survey covers an area of $0.67 \mathrm{deg}^{2}$ in eight pointings $\left(\sim 17^{\prime} \times 17^{\prime}\right.$ each $)$, completely overlapping 
Table 1

Properties of the Data Set in the EGS

\begin{tabular}{|c|c|c|c|c|c|c|}
\hline $\begin{array}{l}\text { Band } \\
\text { (1) }\end{array}$ & $\begin{array}{r}\lambda_{\text {eff }} \\
(2)\end{array}$ & $m_{\lim }[\mathrm{AB}]$ & $\begin{array}{l}\text { FWHM } \\
\text { (4) }\end{array}$ & $\begin{array}{c}\text { Area } \\
(5)\end{array}$ & $\begin{array}{c}\text { Surf. Dens. }\left(\times 10^{3}\right) \\
(6)\end{array}$ & $\begin{array}{c}\text { Source } \\
(7)\end{array}$ \\
\hline Hard X-Ray ${ }^{\text {a }}$ & $0.31 \mathrm{~nm}(2-10 \mathrm{keV})$ & $3.8 \times 10^{-16} \mathrm{erg} \mathrm{cm}^{2} \mathrm{~s}^{-1}$ & $0.5^{\prime \prime}-6^{\prime \prime}$ & 0.67 & 1.102 & Chandra/ACIS; Laird et al. (2009) \\
\hline Soft X-Ray ${ }^{\mathrm{a}}$ & $1.24 \mathrm{~nm}(0.5-2 \mathrm{keV})$ & $5.3 \times 10^{-17} \mathrm{erg} \mathrm{cm}^{2} \mathrm{~s}^{-1}$ & $0.5^{\prime}-4^{\prime \prime}$ & 0.67 & 1.540 & Chandra/ACIS; Laird et al. (2009) \\
\hline FUV & $153.9 \mathrm{~nm}$ & 25.6 & $5^{\prime \prime} .5$ & 1.13 & 10.5 & GALEX GTO \\
\hline NUV & $231.6 \mathrm{~nm}$ & 25.6 & $5 . .5$ & 1.13 & 24.7 & GALEX GTO \\
\hline$u^{\prime}$ & $362.5 \mathrm{~nm}$ & 26.1 & $1^{\prime \prime} 0$ & 0.77 & 148.2 & MMT/Megacam \\
\hline$u^{*}$ & $381.1 \mathrm{~nm}$ & 25.7 & 0.9 & 1 & 152.5 & CFHTLS/MegaCam \\
\hline$B$ & $439.0 \mathrm{~nm}$ & 25.7 & $1^{\prime \prime} .2$ & 1.31 & 101.7 & CFHT-12k \\
\hline$g$ & $481.4 \mathrm{~nm}$ & 26.7 & $1^{\prime \prime} \cdot 3$ & 0.77 & 203.8 & MMT/MegaCam \\
\hline$g^{\prime}$ & $486.3 \mathrm{~nm}$ & 26.5 & 0.9 & 1 & 163.4 & CFHTLS/MegaCam \\
\hline$V_{606}$ & $591.3 \mathrm{~nm}$ & 26.9 & 0.2 & 0.197 & 440.7 & $H S T / \mathrm{ACS}$ \\
\hline$r^{\prime}$ & $625.8 \mathrm{~nm}$ & 26.3 & 0.8 & 1 & 363.9 & CFHTLS/MegaCam \\
\hline$R$ & $651.8 \mathrm{~nm}$ & 26.1 & 0.7 & 1 & 220.0 & Subaru/SuprimeCam \\
\hline$R$ & $660.1 \mathrm{~nm}$ & 25.3 & $1^{\prime \prime} .0$ & 1.31 & 144.3 & CFHT-12k \\
\hline$i^{\prime}$ & $769.0 \mathrm{~nm}$ & 25.9 & 0.8 & 1 & 341.1 & CFHTLS/MegaCam \\
\hline$i$ & $781.5 \mathrm{~nm}$ & 25.3 & $1^{\prime \prime} .0$ & 0.77 & 275.8 & MMT/MegaCam \\
\hline$I$ & $813.2 \mathrm{~nm}$ & 24.9 & $1^{\prime \prime} .1$ & 1.31 & 117.0 & CFHT-12k \\
\hline$i_{814}$ & $807.3 \mathrm{~nm}$ & 26.1 & 0.2 & 0.197 & 452.1 & $H S T / \mathrm{ACS}$ \\
\hline$z^{\prime}$ & $887.1 \mathrm{~nm}$ & 24.7 & 0.8 & 1 & 179.0 & CFHTLS/Megacam \\
\hline$z$ & $907.0 \mathrm{~nm}$ & 25.3 & $1^{\prime \prime} .2$ & 0.77 & 214.0 & MMT/Megacam \\
\hline$J_{110}$ & $1.10 \mu \mathrm{m}$ & 23.5 & 0.7 & 0.0128 & 252.0 & $H S T /$ NICMOS \\
\hline$J$ & $1.21 \mu \mathrm{m}$ & 22.9 & $1^{\prime \prime} .0$ & 0.195 & 67.0 & CAHA- $\Omega 2 k$ \\
\hline$H_{160}$ & $1.59 \mu \mathrm{m}$ & 24.2 & 0.8 & 0.0128 & 252.0 & $H S T / \mathrm{NICMOS}$ \\
\hline$J^{\mathrm{a}}$ & $1.24 \mu \mathrm{m}$ & 21.9 & $1^{\prime \prime}$ & 0.30 & 30.0 & Palomar-WIRC; Bundy et al. (2006) \\
\hline$K_{s}$ & $2.11 \mu \mathrm{m}$ & 20.7 & $1^{\prime \prime} .5$ & 0.20 & 18.6 & CAHA- $\Omega^{\prime}$ \\
\hline$K_{s}$ & $2.15 \mu \mathrm{m}$ & 23.7 & 0.6 & 0.09 & 124.0 & Subaru MOIRCS \\
\hline$K^{\mathrm{a}}$ & $2.16 \mu \mathrm{m}$ & 22.9 & $1^{\prime \prime}$ & 0.70 & 34.0 & Palomar-WIRC; Bundy et al. (2006) \\
\hline IRAC 3.6 & $3.6 \mu \mathrm{m}$ & 23.9 & 2.1 & 0.48 & 315.0 & Spitzer GTO \\
\hline IRAC 4.5 & $4.5 \mu \mathrm{m}$ & 23.9 & 2.1 & 0.48 & 274.4 & Spitzer GTO \\
\hline IRAC 5.8 & $5.8 \mu \mathrm{m}$ & 22.3 & $2 ! .2$ & 0.48 & 129.7 & Spitzer GTO \\
\hline IRAC 8.0 & $8.0 \mu \mathrm{m}$ & 22.3 & 2.2 & 0.48 & 115.2 & Spitzer GTO \\
\hline MIPS 24 & $23.7 \mu \mathrm{m}$ & $19.5(60 \mu \mathrm{Jy})$ & $5^{\prime \prime}$ & 0.79 & 30.0 & Spitzer GTO \\
\hline MIPS 70 & $71.4 \mu \mathrm{m}$ & 15 (3.5 mJy) & $19^{\prime \prime}$ & 0.69 & 6.0 & Spitzer GTO \\
\hline Radio $20 \mathrm{~cm}^{\mathrm{a}}$ & $20 \mathrm{~cm}$ & $100 \mu \mathrm{Jy}_{\text {beam }^{-1}}$ & 4.2 & 0.73 & 1.538 & VLA; Ivison et al. (2007) \\
\hline$R$, redshift ${ }^{\mathrm{a}}$ & $640-910 \mathrm{~nm}$ & 24.1 & $\cdots$ & 1.31 & 10.343 & DEEP2 \\
\hline
\end{tabular}

Notes. Column 1: name of the observing band. Column 2: effective wavelength of the filter calculated by convolving the Vega spectrum (Colina \& Bohlin 1994) with the transmission curve of the filter+detector. Column 3: limiting AB magnitude (except for the X-ray catalogs) of the image estimated as the magnitude of an $\mathrm{S} / \mathrm{N}=5$ detection (see Section 4 for details on the measurement of the photometric errors). Column 4: median FWHM of the PSF in arcseconds measured in a large number of stars (see Section 5.4). Column 5: area covered by the observations in deg ${ }^{2}$. Column 6: source density per square degree up to the limiting magnitude given in Column 3. Column 7: source from where the data were obtained.

a Data drawn from a catalog.

with the region covered by IRAC. The nominal exposure time of the frames is $200 \mathrm{ks}$ per pixel, reaching limiting fluxes of $5.3 \times 10^{-17}$ and $3.8 \times 10^{-16} \mathrm{erg} \mathrm{cm}^{2} \mathrm{~s}^{-1}$ in the soft $(0.5-2 \mathrm{keV})$ and hard $(2-10 \mathrm{keV})$ bands, respectively. In this work, we use the data reduction and point source catalogs published in Laird et al. (2009; see also Nandra et al. 2005 for a first version of the catalogs). The two-band (soft and hard) merged catalog comprises 1325 sources with $<1.5 \%$ spurious detections. The authors identified optical and NIR counterparts for 1013 and 830 sources, respectively, from the CFHTLS optical catalog (see Section 2.3.1), and the Spitzer/IRAC catalog of Barmby et al. (2008). The cross-match is based on maximum likelihood method with a search radius of 2 " (more than a factor of 1.5 the rms of their astrometric accuracy).

\subsection{Ultraviolet Data}

The GALEX (Martin et al. 2005) observed the EGS over three consecutive years providing deep UV data in two channels (FUV at $153 \mathrm{~nm}$, and NUV at $231 \mathrm{~nm}$ ) on a $1.13 \mathrm{deg}^{2}$ circular area around $\alpha=14^{\mathrm{h}} 20^{\mathrm{m}}, \delta=+52^{\circ} 47^{\prime}$. The total exposure times for the composite stacks are $58 \mathrm{ks}$ and $120 \mathrm{ks}$ in the FUV and NUV filters, respectively. The approximate limiting magnitude in both bands is $\sim 25.1 \mathrm{mag}$.

\subsection{Optical Data \\ 2.3.1. CFHTLS/CFHT12K}

A $1^{\circ} \times 1^{\circ}$ square region centered on the coordinates $\alpha=$ $14^{\mathrm{h}} 19^{\mathrm{m}} 27^{\mathrm{s}}, \delta=+52^{\circ} 40^{\prime} 56^{\prime \prime}$ was observed within the CFHTLS (sector D3). Using the MEGACAM camera on the CFHT, imaging data were obtained in five broadband filters over the wavelength range $350 \mathrm{~nm}<\lambda<940 \mathrm{~nm}: u^{*}, g^{\prime}, r^{\prime}, i^{\prime}, z^{\prime}$. The overall exposure times range from $4 \mathrm{hr}$ to $40 \mathrm{hr}$ (for the $u^{*}$ and $z^{\prime}$ bands, respectively), reaching limiting magnitudes between 26 and $27 \mathrm{mag}$. The reduced images have gone through several releases. The data used in this paper are part of the CFHTLS "T0004" release produced at the TERAPIX data center (Gwyn et al. $2011^{6}$ ). Although the total mosaicked area covers $1 \mathrm{deg}^{2}$, the overlap with the IRAC observations is just $0.35 \mathrm{deg}^{2}$.

\footnotetext{
6 http://www.astro.uvic.ca/ gwyn/cfhtls/
} 


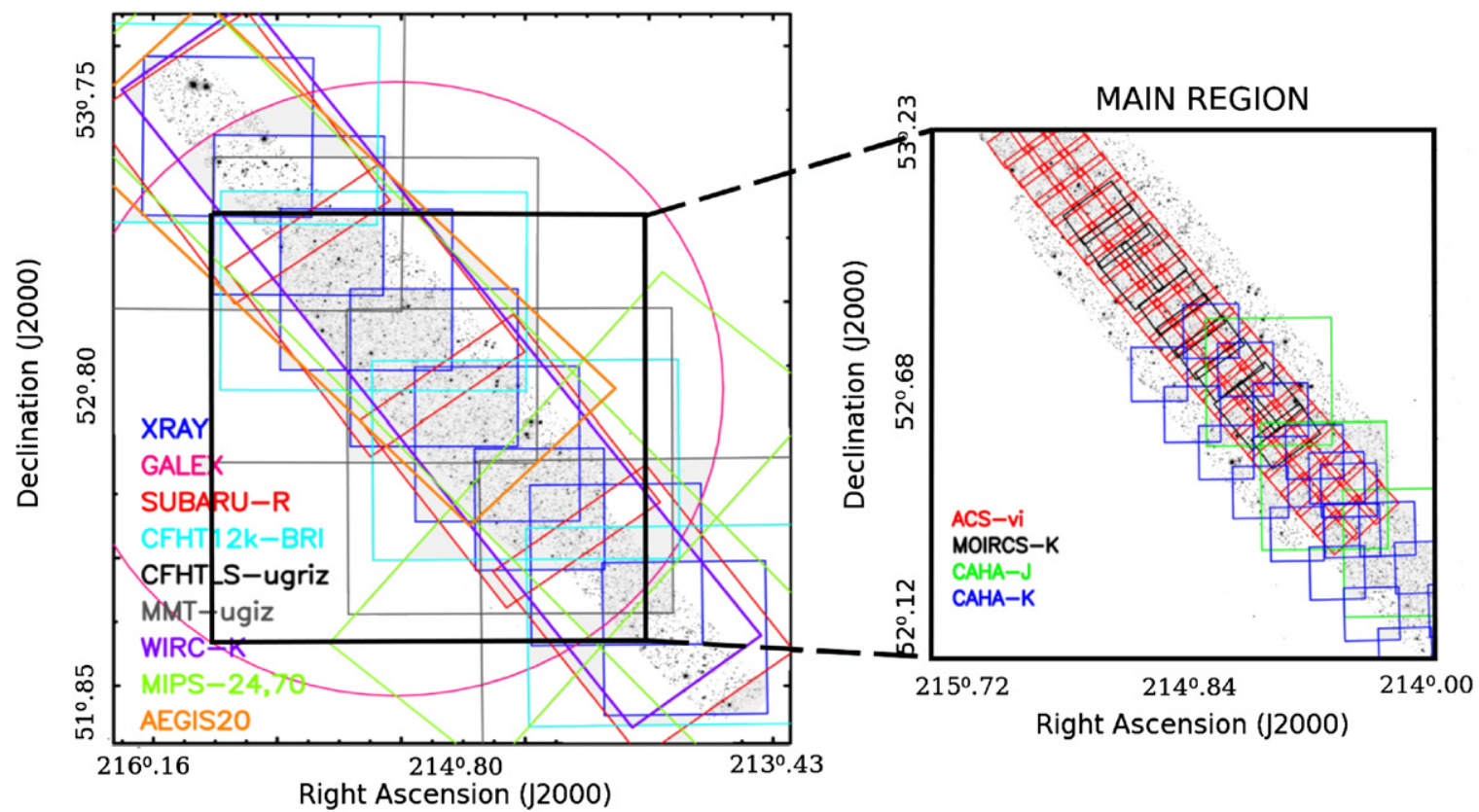

Figure 1. Layout of all the imaging surveys covering the EGS. The background gray-scale image shows the IRAC $3.6 \mu \mathrm{m}$ mosaic. The black square depicts the CFHTLS pointing. The intersection of both footprints defines the region of higher photometric coverage (magnified on the right panel), which is referred as "main region" throughout the text (see Section 5) and contains the highest resolution HST data. The cyan and red rectangles show the CFHT12k-BRI and SUBARU R-band coverage, respectively. The gray and purple squares show the MMT- $u^{\prime}$ giz and Chandra/ACIS pointings, respectively. The magenta circle shows the GALEX survey footprint. The green rectangles show the Spitzer/MIPS coverage. The orange rectangle shows VLA $20 \mathrm{~cm}$ coverage. In the main region, magnified on the right side of the figure, the red tiles depict the HST/ACS footprints (the NICMOS images overlap with this area). Black rectangles show the MOIRCS $K_{s}$-band imaging, and the green and purple lines delimit the CAHA $J$ - and $K_{s}$-band surveys.

In addition to the CFHTLS data, shallower images were acquired in the $B, R$, and $I$ bands over a larger area, using the wide-field $12 \mathrm{~K}$ mosaic camera on CFHT. These observations were intended to provide optical coverage for the DEEP2 spectroscopic survey, extending through the whole EGS in four different pointings. The observing times were $3-6 \mathrm{hr}$, to achieve a limiting magnitude of $\sim 25$ mag. The photometric catalogs were published within the DEEP2 DR1 (Coil et al. 2004), and the raw (non-reduced) images can be retrieved from the CADC archive. ${ }^{7}$ We downloaded the raw and calibrations files from the archive to perform our own data reduction and cataloging. This reduction was carried out with the $\mathrm{IRAF}^{8}$ task mscred. The photometric calibration was performed using the $B R I$ public catalogs. The average dispersion of the photometry comparison is smaller than $0.03 \mathrm{mag}$.

\subsubsection{MMT/Megacam}

The EGS was observed with the Megacam camera (McLeod et al. 2006) on the MMT in the $u^{\prime}$ giz bands during several campaigns from 2006 to 2009 . Four pointings of the $24^{\prime} \times 24^{\prime}$ Megacam field of view were arranged to cover the full extension of the 2 deg long IRAC mosaic.

The MMT/Megacam imaging data were reduced with a combination of standard IRAF/mscred routines and custom software. Final mosaics were calibrated against the coextensive SDSS photometry with appropriate color corrections. The $5 \sigma$ limiting magnitudes within a $2^{\prime \prime}$ diameter aperture for point sources vary among the four different pointings but are approximately 26.5, 27.2, 26.0, and $26.0 \mathrm{mag}$ in the $u^{\prime}, g, i$, and $z$ bands,

\footnotetext{
7 http://www4.cadc-ccda.hia-iha.nrc-cnrc.gc.ca/cadcbin/cfht/wdbi.cgi/ cfht/wdbi.cgi/cfht/quick/form

8 http://iraf.noao.edu/
}

respectively. The effective seeing in the final mosaics is roughly $1^{\prime \prime}$ FWHM or slightly better in some bands.

\subsubsection{HST/ACS}

As a part of a GO program (PI: Davis), the $H S T$ Advanced Camera for Surveys (ACS) acquired deep imaging of EGS in two optical bands: $F 606 W$ and $F 814 W$ (hereafter the $V_{606}$ and $i_{814}$ images). The ACS survey covers an area of $\sim 710.9 \mathrm{arcmin}^{2}$ $\left(10^{\prime} 1 \times 70\right.$ '.5) on 63 contiguous tiles following the direction of the IRAC mosaic, covering approximately $50 \%$ of the total area surveyed by IRAC, and $\sim 80 \%$ of the area overlapping with the CFHTLS observations. The fully reduced, drizzled frames and calibration products were released by the AEGIS Team (Lotz et al. 2008). Each science image has an approximate exposure time of $\sim 0.6 \mathrm{hr}$ per pixel and a limiting magnitude of $\sim 28 \mathrm{mag}$.

\subsubsection{Subaru Suprime-Cam}

Ground based $R$-band imaging of the EGS was carried out with the Subaru Telescope as a part of the Subaru Suprime-Cam Weak-Lensing Survey (Miyazaki et al. 2007). The SuprimeCam field of view is $0.25 \mathrm{deg}^{2}$ in size, covering the whole IRAC map in four pointings. The total exposure time was 30 minutes for each pointing, taken in four 7.5 minute exposures, in a dithering pattern with $\sim 1^{\prime}$ spacing. The approximate limiting magnitude is $R=26 \mathrm{mag}$. These data were downloaded from the SMOKA database and reduced with the Suprime-Cam pipeline (SDFRED, v1.0).

\subsection{Near-infrared Data}

\subsubsection{HST/NICMOS}

Simultaneously to the ACS observations, the Near-Infrared Camera and Multi-Object Spectrometer (NICMOS) covered 
parallel fields in the $F 110 W$ and $F 160 W$ bands (hereafter $J_{110}$ and $H_{160}$ bands, respectively), with a similar exposure time and a limiting magnitude of 23.5 and 24.2, respectively. The observations were designed to maximize the overlap between both HST surveys. Virtually all (58 out of the 63) NICMOS frames lie within the area covered by the ACS primary imaging. However, the smaller field of view of NICMOS $\left(\sim 1^{\prime} \times 1^{\prime}\right)$ leads to a total NIR coverage of only $0.0128 \mathrm{deg}^{2}$, with a $90 \%$ overlap with the area covered by ACS but less than a $4 \%$ with that covered by IRAC.

\subsubsection{Subaru MOIRCS}

In addition to the optical imaging, NIR observations of the EGS were also obtained with the Multi-Object InfraRed Camera and Spectrograph (MOIRCS) on five nights during 2006 April-May (PIs: Fukugita, Yamada) and a complementary run on 2007 June 25 . The data set comprises 11 pointings covering a total of $0.09 \mathrm{deg}^{2}$ oriented along the original strip within $52.5<\delta<53.0$ and completely overlapping with the HST-ACS and CFHTLS imaging. The median exposure time per frame is $\sim 1 \mathrm{hr}$ for an approximate limiting magnitude of $K_{s} \sim 23-24 \mathrm{mag}$. The data were reduced using dedicated scripts developed by the MOIRCS team involving the IRAF task MSCRED, plus an additional de-fringing process (see, e.g., Kajisawa et al. 2009).

\subsubsection{Palomar and Calar Alto Imaging}

Given the importance of having a continuous band coverage for any kind of study regarding galaxy populations (Wuyts et al. 2007), we have incorporated in our data compilation the POWIR NIR catalog (Bundy et al. 2006). These data were acquired between 2002 September and 2005 October using the WIRC camera in the Palomar $5 \mathrm{~m}$ telescope. The total surveyed area in the EGS field is $2165 \operatorname{arcmin}^{2}\left(0.6 \mathrm{deg}^{2}\right)$ in the $K$ band and $\sim 1 / 3$ of that area in the $J$ band. The approximate limiting magnitudes are $K \sim 22.9$ and $J \sim 21.9$. Note that no images were publicly available for this data set, so we only use the catalogs.

In addition, we also make use of the NIR imaging obtained by the Galaxy evolution and Young Assembly $\left(\mathrm{GOYA}^{9}\right)$ project. Two photometric campaigns were carried out to obtain NIR data of the original Groth Strip $\alpha=14^{\mathrm{h}} 17^{\mathrm{m}} 43^{\mathrm{s}}, \delta=52^{\circ} 28^{\prime} 41^{\prime \prime}$ (Cristóbal-Hornillos et al. 2003) and flanking fields (Barro et al. 2009). Here we make use of the $K$-band images of the flanking fields, observed with the $\Omega^{\prime}$ instrument in the $3.5 \mathrm{~m}$ telescope at Calar Alto Spanish-German Astronomical Center (CAHA). The frames cover a total area of $\sim 0.24 \mathrm{deg}^{2}$ to a limiting magnitude of $K_{s} \sim 20.7$.

Finally, we have also included data in the $J$ band from the narrowband survey of $\mathrm{H} \alpha$ emitters described in Villar et al. (2008). Three $15^{\prime} \times 15^{\prime}$ pointings centered at $\alpha=14^{\mathrm{h}} 17^{\mathrm{m}} 31^{\mathrm{s}}$, $\delta=52^{\circ} 28^{\prime} 11^{\prime \prime}, \alpha=14^{\mathrm{h}} 17^{\mathrm{m}} 31^{\mathrm{s}}, \delta=52^{\circ} 28^{\prime} 11^{\prime \prime}$ and $\alpha=$ $14^{\mathrm{h}} 18^{\mathrm{m}} 14^{\mathrm{s}}, \delta=52^{\circ} 42^{\prime} 15^{\prime \prime}$ were observed in CAHA using the $\Omega 2 k$ instrument in the $3.5 \mathrm{~m}$ telescope. The combined pointings cover an area of $0.19 \mathrm{deg}^{2}$ to a limiting magnitude of $J=22.9$.

\subsection{Mid-to-far IR Data}

\subsubsection{Spitzer/IRAC}

Our sample is drawn from Spitzer near/mid-IR data obtained as part of the Guaranteed Time Observations (GTO; PI: Fazio) and presented in Barmby et al. (2008). We also included

\footnotetext{
9 http://www.astro.ufl.edu/GOYA/home.html
}

additional data from the GO program with ID 41023 (PI: K. Nandra). The GTO IRAC imaging data at 3.6, 4.5, 5.8, and $8.0 \mu \mathrm{m}$ were obtained over two epochs (2003 December and 2004 June/July). The data set comprises 52 different pointings that cover a $2^{\circ} \times 10^{\prime}$ strip with approximately the same depth. To achieve this homogeneous coverage and due to scheduling issues, the width of the mosaic is slightly variable along the strip, ranging from $10^{\prime}$ to $17^{\prime}$. The average exposure time per pixel is $2.5 \mathrm{hr}(9100 \mathrm{~s})$ in the four channels. An area of $1440 \mathrm{arcmin}^{2}$ was observed for $1900 \mathrm{~s}, 930 \mathrm{arcmin}^{2}$ for $9100 \mathrm{~s}$, and $\sim 100 \mathrm{arcmin}^{2}$ for $>11,500 \mathrm{~s}$ (Barmby et al. 2008). The additional GO data are located in two strips of width $\sim 3.5$ flanking the original strip and covering the declination range 52.35 $<\delta<53.25$. All the data were reduced with the general Spitzer pipeline, which provides Basic Calibrated Data, and then mosaicked with Mopex using a pixel scale half of the original $\left(\sim 0.61 \operatorname{arcsec}_{\text {pixel }}^{-1}\right)$. The details of the image quality are discussed in Section 3.2.

\subsubsection{Spitzer/MIPS $24 \mu \mathrm{m}$ and $70 \mu \mathrm{m}$}

Complementary to the IRAC observations, MIR and FIR observations were also obtained with the Multiband Imaging Photometer for Spitzer (MIPS; Rieke et al. 2004) as part of the GTO and the Far-Infrared Deep Extragalactic Legacy Survey (FIDEL). For this paper, we use the whole GTO+FIDEL data set, reduced and mosaicked with the Spitzer pipeline and MOPEX+GeRT software. The surveyed area covers approximately the entire $2^{\circ} \times 10^{\prime}$ strip, being slightly wider on the upper and lower edges. The MIPS mosaic overlaps with the deepest part of the IRAC observations. The mean exposure time at $24 \mu \mathrm{m}$ is $\sim 7200 \mathrm{~s}$ per pixel, while for the $70 \mu \mathrm{m}$ channel it is approximately $3800 \mathrm{~s}$. The approximate limiting fluxes are $60 \mu \mathrm{Jy}$ and $3.5 \mathrm{mJy}$, respectively.

\subsection{Radio Data}

A radio survey at $1.4 \mathrm{GHz}(20 \mathrm{~cm})$ of the northern half of the EGS ( $\sim 50 \%$ of the IRAC mosaic) was conducted with the Very Large Array (VLA) in its B configuration during 2003-2005. The AEGIS20 survey covers $0.73 \mathrm{deg}^{2}$ down to $130 \mu \mathrm{Jy} \mathrm{beam}^{-1}$ including a smaller region of $0.04 \mathrm{deg}^{2}$ with a $50 \mu \mathrm{Jy}$ detection limit $(5 \sigma)$. The data reduction and the source catalog, comprising 1123 sources, were presented in Ivison et al. (2007).

\subsection{Keck Optical Spectra}

EGS has also been the target of an exhaustive and unique spectroscopic follow-up. As one of the DEEP2 fields, optical multi-object spectroscopy has been carried out from 2003 to 2005 with the Deep Imaging Multi-Object Spectrograph (DEIMOS; Faber et al. 2003) on the Keck II telescope. The observations cover the spectral range $640 \mathrm{~nm}<\lambda<910 \mathrm{~nm}$ with a resolution of $0.14 \mathrm{~nm}$. The DEEP2 DR3 ${ }^{10}$ contains spectroscopic redshifts for 13,867 sources at $0<z<1.4$ with a median redshift $z=0.75$. The targets were selected from the CFHT12k-BRI images (within $1.31 \mathrm{deg}^{2}$ ) in the magnitude range $18.5 \leqslant R \leqslant 24.1$. The spectroscopic redshifts for around $70 \%$ of the sources are labeled with a high-quality flag (values of 3 and 4 , meaning $>95 \%$ success rate). Lower quality flags are considered unreliable and will be excluded from our analysis here and in Paper II.

\footnotetext{
${ }_{10}$ http://deep.berkeley.edu/DR3/dr3.primer.html
} 
We complemented the DEEP2 spectroscopy of $z<1.4$ galaxies with redshifts for $z \sim 3$ sources from the Lyman Break Galaxy (LBG) survey of Steidel et al. (2003). This survey covers a total area of $0.38 \mathrm{deg}^{2}$ divided in several fields, one of them centered in the EGS. The observations in EGS consist of a single $15^{\prime} \times 15^{\prime}$ mask centered at $\alpha=14^{\mathrm{h}} 17^{\mathrm{m}} 43^{\mathrm{s}}$, $\delta=52^{\circ} 28^{\prime} 48^{\prime \prime}$ observed with the Low Resolution Imaging Spectrometer (LRIS; Oke et al. 1995) on Keck. The spectra cover the 400-700 $\mathrm{nm}$ range with a median resolution of $0.75 \mathrm{~nm}$. The targets were pre-selected based on the LBG color-color criteria (Steidel et al. 1996) including only candidates brighter than $R=25.5$. The EGS catalog contains a total of $334 \mathrm{LBG}$ candidates in the surveyed area. Out of them, 193 are spectroscopically confirmed to be at $z \sim 3$. Unfortunately, the overlap with the IRAC frame is not complete (and some of the galaxies are extremely faint in the IRAC bands), and we were only able to identify 243 ( $72 \%$ of the spectroscopic sample) LBGs in our $3.6+4.5 \mu \mathrm{m}$ selected catalog (we give more details on these sources in Paper II).

\section{DESCRIPTION OF THE SAMPLE SELECTION}

The data set described in the previous section was used to obtain UV-to-FIR SEDs for all the sources detected in the EGS IRAC survey. This merged photometric catalog was built following the procedure described in PG05 and PG08. Here we review all the basic steps of the method, emphasizing the improvements introduced for this paper concerning the extraction of the IRAC catalog and the band merging procedure.

\subsection{The IRAC $3.6 \mu \mathrm{m}+4.5 \mu \mathrm{m}$ Selection}

The source detection in the IRAC data was carried out separately in the $3.6 \mu \mathrm{m}$ and $4.5 \mu \mathrm{m}$ images using SExtractor (Bertin \& Arnouts 1996). The complementary detection in the slightly shallower $4.5 \mu \mathrm{m}$ band helps to alleviate the source confusion problems arising from the point-spread function (PSF) size and the remarkable depth of the IRAC data. Both catalogs were cross-matched using a $1^{\prime \prime}$ search radius to remove repeated sources. This produces a master IRAC-selected catalog containing the sources detected in any of the two channels. Eventually, most sources are simultaneously detected in both channels.

The average survey depth is remarkably homogeneous across the strip, $t_{\exp } \sim 10 \mathrm{ks}$, with the exception of two small areas with lower exposure at the top $(\delta>53.525)$ and bottom $(\delta<52.025)$ of the mosaic. We took into account the lower exposure times near the edges of the images by defining two different areas: a shallower region with exposure time shorter than $3800 \mathrm{~s}$ ( $N$ (frames $) \leqslant 20)$, and a deeper region covering the majority of the strip. The detection was carried out with different SExtractor parameters in each region, using a more conservative configuration for the shallower region. Then, we used this (more restrictive) catalog to purge some low significance detections in the other catalog within an overlapping area between them $(N$ (frames $)=18-25)$. The purged catalog restricted to the area with $N$ (frames) $>20$ constitutes our master photometric catalog, and it covers an area of $0.50 \mathrm{deg}^{2}$.

After the detection of sources, we removed spurious sources in the wings of bright stars (where the PSF shows bright knots). For that purpose, first we made a preliminary detection of star-like sources based on the IRAC color-color criteria of Eisenhardt et al. (2004; see Section 5.4). Then, we eliminated detections within the typical distance where the contamination from the star is significant (typically $\sim 9^{\prime \prime}$ for the typical star magnitudes and depth of the EGS observations). After masking the regions around stars, the total area covered by the catalog is $0.48 \mathrm{deg}^{2}$.

Aperture photometry for all $3.6 \mu \mathrm{m}+4.5 \mu \mathrm{m}$ detected sources was measured with our own dedicated software (which takes into account pixel fractions appropriately; see PG08) in all four IRAC images, previously registered to the same World Coordinate System (WCS). The flux is measured in the four IRAC bands simultaneously. If a source is undetected in the shallower bands (i.e., [5.8] and [8.0]) we still measure an upper limit flux as three times the rms of the sky. The majority of the IRAC sources are unresolved in the $3.6 \mu \mathrm{m}$ image (FWHM $\sim 2^{\prime \prime}$ ). However, most of them are not point-like, but slightly extended. Consequently, PSF fitting is not effective and photometry is best measured with small circular apertures (PG08; Barmby et al. 2008; Wuyts et al. 2008; Ilbert et al. 2009). The flux measurement in all bands was carried out at the positions specified in the IRAC master catalog. We used a $2^{\prime \prime}$ radius aperture and applied aperture corrections for each band derived from the PSF growth curves. The values of the correction are $[0.32 \pm 0.03,0.36 \pm 0.02,0.53 \pm 0.02$, $0.65 \pm 0.02] \mathrm{mag}$ at $[3.6,4.5,5.8,8.0] \mu \mathrm{m}$. The errors account for the typical WCS alignment uncertainties. For a small number of extended sources $(\sim 2 \%$ of the total catalog, and $75 \%$ of them presenting [3.6] $<22.3)$, the $2^{\prime \prime}$ aperture tend to underestimate the total magnitudes (by more than a 10\%). These sources are typically bright nearby galaxies whose Kron (1980) radius is larger than $\sim 4$.'5. The flux measurement for these sources was performed in larger apertures enclosing the full object and applying the extended source aperture corrections given in the Spitzer/IRAC cookbook.

The uncertainties in the IRAC photometry were computed taking into account the contributions from the sky emission, the readout noise, the photon counting statistics, the uncertainties in the aperture corrections, and a $2 \%$ uncertainty from the zeropoint absolute calibration (Reach et al. 2005). We did not assume the uncertainties resulting from SExtractor flux measurement. Instead, we used a more realistic method to determine the background noise that takes into account the effects of pixel-topixel signal correlation. This procedure has also been applied to measure the photometric uncertainties in all the bands and is outlined in Section 4.3. Nevertheless, a straightforward comparison to the SExtractor errors indicates that the noise correlation does not introduce a significant contribution to the flux uncertainties at bright magnitudes, leading to a median increment $\leqslant 0.02$ mag up to $[3.6,4.5] \sim 23.75$.

A detailed comparison of our IRAC catalog of the EGS survey to the one published by Barmby et al. (2008) is presented in Section 3.3, including a discussion on the source confusion levels.

\subsection{Completeness and Limiting Magnitude of the IRAC Catalog}

We estimated the completeness of the IRAC catalog by analyzing the recovery of simulated sources added in the mosaicked images. The simulations were carried out in the central regions of the mosaic where the coverage is uniform $\left(t_{\exp } \geqslant 10 \mathrm{ks}\right)$. Artificial sources spanning a wide range of sizes (from $1^{\prime \prime}$ to $6^{\prime \prime}$ ) and brightnesses ([3.6] $=16-25 \mathrm{mag}$ ) were created on the IRAC images at random locations. The number of simulated sources was chosen to be representative 

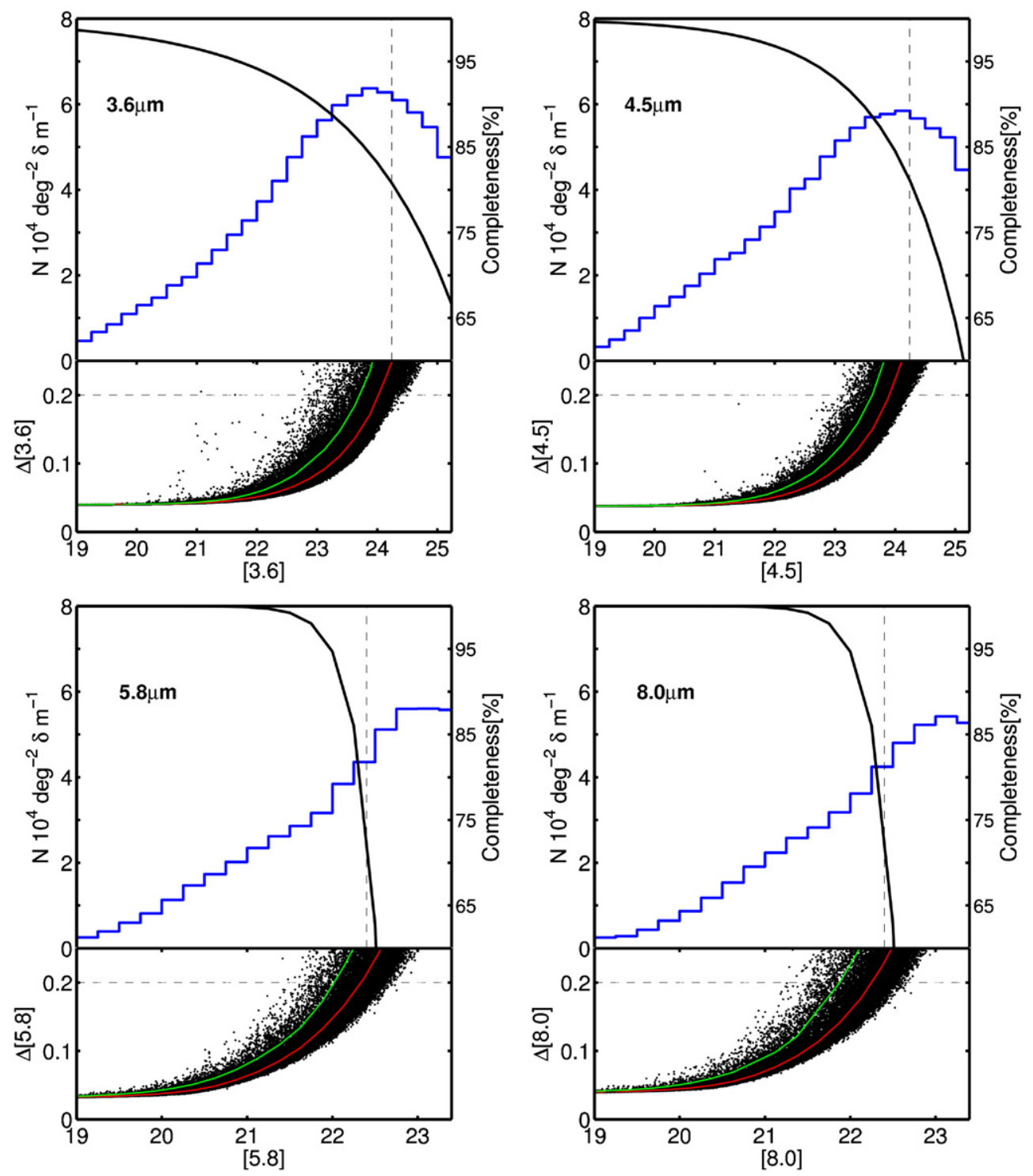

Figure 2. Upper panel of each quadrant shows the histogram of IRAC magnitudes for the sources in our sample selected in the IRAC $3.6 \mu \mathrm{m}+4.5 \mu \mathrm{m}$ data. The histograms are shown up to the $2 \sigma$ limiting magnitude in each band. The blacks continuous curve depicts the detection efficiency as estimated from recovery rate of simulated punctual sources. The vertical dashed lines represent five times the median sky rms $(5 \sigma)$ measured in a large number of $2^{\prime \prime}$ radius apertures. The lower panels in each quadrant show the distribution of photometric uncertainties as a function of the magnitude in each IRAC band. The red and green lines indicate the median and $90 \%$ of the error distribution as a function of magnitude, respectively. The horizontal dashed line shows the S/N $\sim 5$ limit; the intersection with the red line indicates the values quoted in Table 1 for the IRAC bands. Note that these values are slightly lower than the $5 \sigma$ sky rms.

of the Poisson uncertainty in the observed number densities. The source detection and photometry was performed again in the simulated images keeping the same SExtractor parameters as in the original frames. The success rate recovering the simulated sources allows us to estimate the completeness level as a function of magnitude. Figure 2 summarizes the completeness analysis in the four channels. For simplicity, we show results only for point-like sources. The completeness for extended sources is typically $\sim 10 \%$ lower at faint magnitudes, however, these sources represent a very small fraction of the catalog at these magnitudes. Figure 2 also shows the source density as function of the magnitude in each band. We find that the catalog is $85 \%$ complete for point sources with $[3.6,4.5]=23.75 \mathrm{mag}$ and $75 \%$ complete at $[3.6,4.5]=24.75 \mathrm{mag}$. In the two other channels, the detection efficiency is significantly lower, with $85 \%$ completeness at $[5.8,8.0]=22.25$, and the completeness dropping rapidly beyond that magnitude. Note that the forced photometric measurement in these bands provides a significant number of $<5 \sigma$ (vertical dashed lines in Figure 2) detections that would be missed otherwise.

The lower panels of Figure 2 show the photometric uncertainties as a function of magnitude in the four IRAC bands. The red and green lines indicate the median and the level enclosing $90 \%$ of the distribution, respectively. For the region with the deepest coverage, we estimated a $3 \sigma$ limiting magnitude of [3.6,4.5] $\sim 24.75$ and $[5.8,8.0] \sim 22.90$ from the median value of the sky rms in our default photometric apertures ( 2 " radius).

The 3.6+4.5 $\mu \mathrm{m}$ catalog contains 70,048 and 99,618 sources with [3.6] $<23.75$ and [3.6] $<24.75$, respectively. The median magnitude of the sample up to [3.6] $<24.75$ is $[3.6]=23.14$, and $75 \%$ of the sources present $[3.6]<23.96$. Note that these numbers correspond to the IRAC $3.6+4.5 \mu \mathrm{m}$ catalog before applying the deblending technique discussed in Section 5. Consequently, the number of sources quoted above is lower than in the final catalog (see Table 2). 
Table 2

Number of Sources in the IRAC $3.6+4.5 \mu \mathrm{m}$ Catalog

\begin{tabular}{|c|c|c|}
\hline Sample & {$[3.6] \leqslant 23.75^{\mathrm{a}}$} & {$[3.6] \leqslant 24.75^{\mathrm{b}}$} \\
\hline Prior to deblending & 70,048 & 99,618 \\
\hline After deblending & $76,936^{\mathrm{c}}$ & $113,023^{\mathrm{d}}$ \\
\hline Main $^{\mathrm{e}} /$ Flanking regions & $53,030 / 23,906$ & $77,607 / 35,416$ \\
\hline
\end{tabular}

Notes.

a $85 \%$ completeness level.

b $3 \sigma$ limiting magnitude.

c Only these sources have been included in the final catalog presented in Section 6.

d A larger, although less complete, sample including all the sources down to [3.6] $<24.75$ can be accessed through the Web interface Rainbow Navigator (Section 6.3).

e The main region is defined as: $52.16<\delta<53.20$ and $214.04<\alpha<215^{\circ} .74$. The flanking regions consist of the remaining area.

\subsection{Comparison to Barmby et al. IRAC-selected Catalog}

Here we compare our IRAC photometric catalog to that published by Barmby et al. (2008, hereafter BAR08). BAR08 used the same data set (except for the GO flanking regions) and obtained final mosaics in all four IRAC bands with a very similar reduction to ours. Concerning the source detection, our method is slightly different from BAR08 since we detect galaxies in both the 3.6 and $4.5 \mu \mathrm{m}$ channel instead of only using the bluer band. Our dual detection technique helps to alleviate the source confusion problems arising from the PSF size and the remarkable depth of the IRAC data. In addition, we measure fluxes in the four channels simultaneously, obtaining upper limit values for undetected sources in the shallower bands ([5.8] and [8.0]). Note also that we have increased the resolution of our catalog by deconvolving blended sources using higher resolution information from ground-based observations (Section 4.1). However, for the sake of clarity, we compare here the BAR08 catalog with ours before carrying out the deblending procedure.

BAR08 measured aperture photometry with SExtractor. The publicly available catalog ${ }^{11}$ includes MAG_AUTO and MAG_ISO measurements, jointly with aperture magnitudes for several radii corrected to total magnitudes with empirical PSF corrections. We compare our photometry to the magnitudes measured in the 3.5 pixel aperture $\left(2{ }^{\prime \prime} 1\right)$ by BAR08. Their aperture corrections agree with our measurements for the 4 " diameter aperture within the errors (due to alignment uncertainties).

BAR08 and our catalog are cross-correlated in the region of highest exposure $\left(t_{\exp }>4 \mathrm{ks}\right)$ using a $1^{\prime \prime}$ radius. The comparison of the WCS between the two mosaics is in very good agreement, with an rms of $<0$ '.05. We find 41,514 and 52,130 sources in common up to [3.6] $<23.75$ and [3.6] $<24.75$, respectively.

Attending to the density of sources per unit area, we find that our catalog includes $11 \% \pm 5 \%$ and $18 \% \pm 7 \%$ more sources than the catalog published by BAR08 at [3.6] $<23.75 \mathrm{mag}$ and [3.6] $<24.75 \mathrm{mag}$, respectively. The uncertainties in these measurements were estimated by comparing number counts in $0.5 \mathrm{mag}$ bins, including Monte Carlo simulations on the photometric errors.

The different source densities are a consequence of their more conservative SExtractor detection threshold. We have carefully chosen the SExtractor parameters differentiating between the regions with high/low coverage (Section 3.1), trying to push down

\footnotetext{
11 http://www.cfa.harvard.edu/irac/egs/
}

the detection limits as much as possible without degrading the reliability of the entire catalog. As a consequence, our catalog recovers a larger number of sources at fainter magnitudes, and the completeness of our catalog is larger than BAR08 for the same magnitude. For example, they quoted a $\leqslant 50 \%$ completeness for point-like sources at [3.6] $=23.75 \mathrm{mag}$, compared to our estimated $85 \%$. Unfortunately, lowering the detection threshold inevitably increases the number of spurious detections. However, in the context of a merged multi-band photometric catalog, spurious detections can be efficiently identified as sources only detected by IRAC (cf. $N$ (band) $<5$ ), and we will show that the reliability is $>97 \%$, with false detections located almost uniquely around very bright sources (see Section 5.3).

Figure 3 shows the comparison of the photometric magnitudes in the four bands for both catalogs. In both cases, the flux was measured on circular apertures and corrected to total magnitudes using aperture corrections. We have corrected the comparison by a constant value of $-0.05 \mathrm{mag}$ in [3.6] and [4.5] and by $-0.04 \mathrm{mag}$ and $-0.03 \mathrm{mag}$ in [5.8] and [8.0], respectively. Such small offsets are attributed to slight differences in the data reduction (final absolute calibration, frame stacking, registering, and mosaicking) and in the aperture corrections. Despite the small offsets, the overall results in the four IRAC bands are in good agreement. Note that the average photometric error in our catalog for each magnitude bin (green bars) encloses $1 \sigma$ of the values around the median difference (red and cyan lines).

The lower panel of each plot in Figure 3 shows the comparison of the photometric errors in BAR08 and in our catalog. Our quoted photometric uncertainties tend to differ from BAR08, specially at faint magnitudes. In contrast with that paper, we have considered zero-point and WCS uncertainties, resulting in slightly larger uncertainties $(\sim 0.05 \mathrm{mag})$ in our catalog for bright sources up to [3.6][ 4.5] $\sim 21 \mathrm{mag}$ and [5.8][ 8.0] $\sim$ $19 \mathrm{mag}$. At fainter magnitudes, the photometric uncertainties increase with magnitude at a faster rate in the catalog of BAR08. The cause for this difference is the procedure to measure the background noise. Similarly to BAR08, we estimated this value from the sky variance measured in circular apertures at different locations of the images that are empty of sources (Section 4.3). However, the definition of an empty region depends on the limits of source detection. Therefore, given our higher detection fraction, our sky regions would contain, in principle, lower signal pixels effectively decreasing the rms. In addition, our photometric procedure estimates uncertainties on a source-by-source basis studying the background around each object in a independent way, while BAR08 relied on SExtractor photometric errors and applied a correction to them based on the average properties of the mosaic. Nevertheless, our estimates of the photometric errors are consistent with the observed scatter of the comparison between our photometry and that measured by BAR08.

\section{MULTI-WAVELENGTH PHOTOMETRY: THE RAINBOW CATALOG}

Using the whole data set available in the EGS field, we created a multi-wavelength photometric catalog for the IRAC-selected sample described in the previous section. For that purpose, we used the Rainbow software package, described in detail in PG05 and PG08. This software was created to: (1) cross-correlate multi-band catalogs and obtain consistent (aperture matched) photometry on the different bands to build a UV-to-FIR SED and (2) estimate stellar parameters, such as photometric redshifts, stellar masses, and SFRs from those SEDs. In the rest of this 

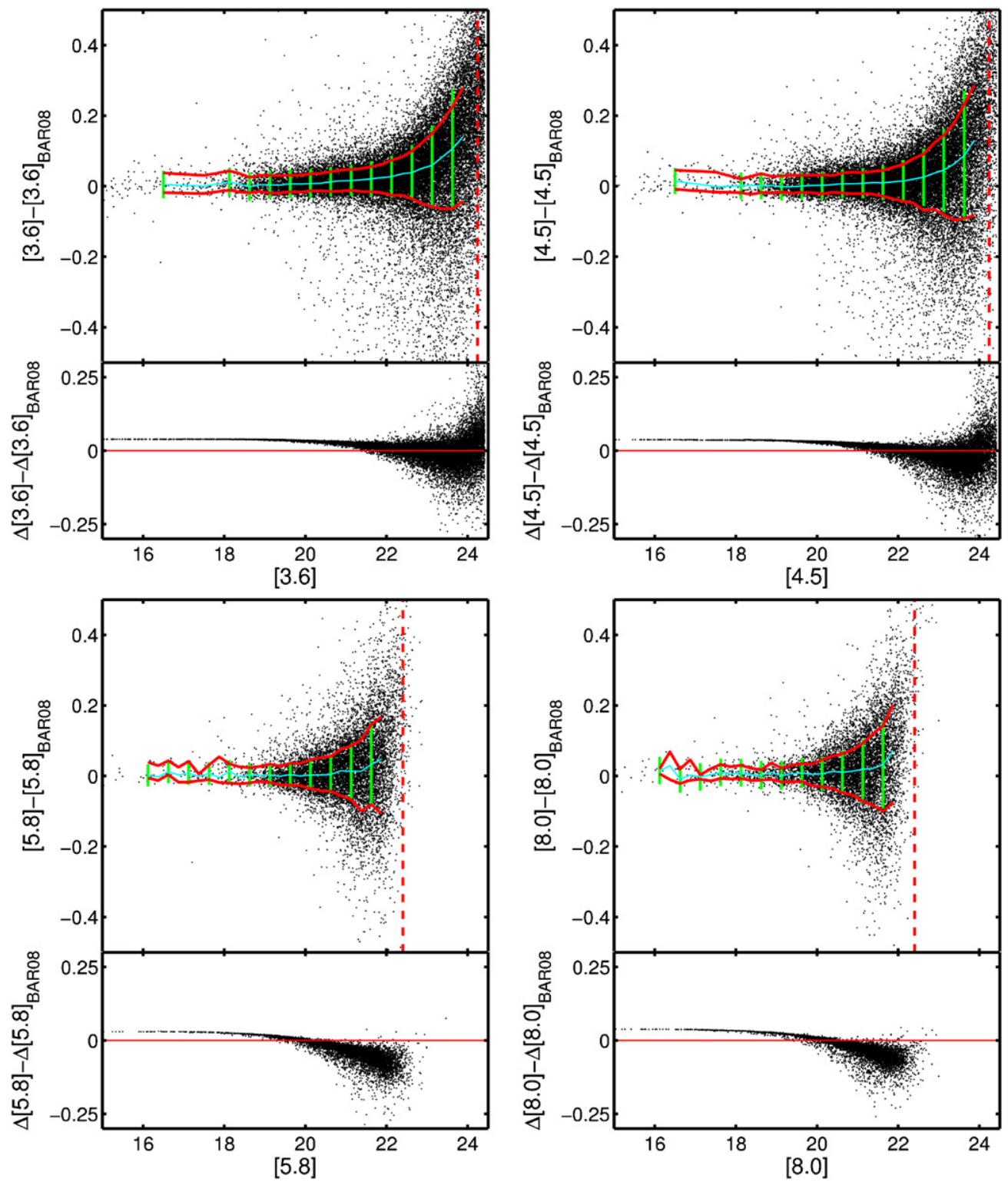

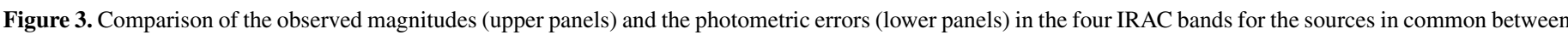

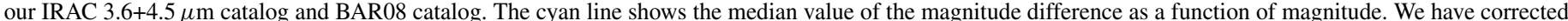

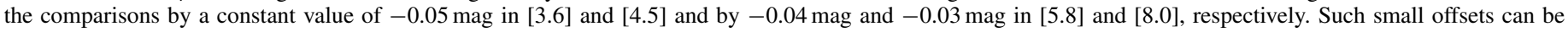

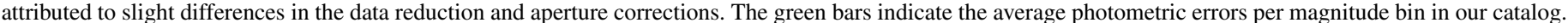

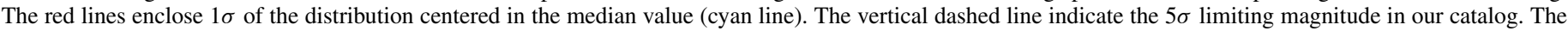

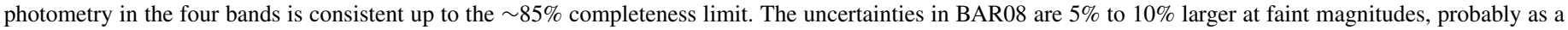
result of the slightly different procedure applied to measure the sky background.

section we will describe the photometry procedure, and in Paper II we will present the methods to estimate photometric redshifts, stellar masses, and SFRs out the SEDs.

\subsection{Cross-correlation and Source Deblending}

The Rainbow code starts from a primary selection catalog (in our case, IRAC selected) and obtains merged photometry and spectroscopy in other bands. The first step is to identify the counterparts of the IRAC sources in the other bands, where SExtractor catalogs have been built following typical procedures. These catalogs are cross-correlated to the $3.6+4.5 \mu \mathrm{m}$ positions using a $2^{\prime \prime}$ search radius. An exception to this rule are the MIPS, radio, and X-ray catalogs. For the MIPS and radio bands, we used a 2 . 5 and $3^{\prime \prime}$ radius, respectively, recognizing possible alignment and center estimation problems of the order of one pixel. For the latter, instead of using the WCS of the X-ray sources, we cross-matched to the positions of the IRAC counterparts (given in Laird et al. 2009) using a $1^{\prime \prime}$ radius. These authors used as reference the IRAC catalog of BAR08, which covers a slightly lower area than ours. Thus, for sources outside of the BAR08 mosaic we cross-matched to the X-ray coordinates using a $2^{\prime \prime}$ radius (see Section 5.2 for more details).

The IRAC sources are identified in this way with objects in all the other catalogs. One of the optical catalogs (typically the deepest; in the EGS, the Subaru $R$-band data) is used as a reference to narrow the following cross-correlations and alleviate confusion problems present in the IRAC images. As the cross-correlation to the catalog of spectroscopic redshifts is done to the coordinates of the counterpart in the reference (optical) band, it is possible to choose a more reliable 0 '.75 search radius for this catalog. 
Before the cross-matching procedure is carried out between a pair of images, these are re-aligned locally within a $4^{\prime} \times 4^{\prime}$ square region using the positions of several sources (typically more than $20)$ as reference. The mean rms between the central coordinates of matched sources in optical/NIR images is typically $\leqslant 0$.' 1 and $\leqslant 0^{\prime} .2$ between the IRAC and the ground-based images. This procedure allows to overcome small misalignment problems between the frames and assures a reliable identification of counterparts and an accurate positioning of the photometric aperture in all bands. It also allows to obtain reliable photometry (in the appropriate aperture) even if the source is very faint and/or undetected in an individual image.

The combination of the remarkable depth and the $\sim 2^{\prime \prime}$ FHWM of the IRAC observations inevitably leads to issues of source confusion, specially around crowded environments. However, based on the (ground-based) reference image, it is possible to deblend IRAC sources which have not been separated by SExtractor in the original IRAC images and lie at least $1^{\prime \prime}$ away (half the FWHM, chosen as our resolution criterion). HST images reveal that the multiplicity is larger, but the deconvolution of sources separated by less than $1^{\prime \prime}$ is very uncertain. When multiple counterparts are found in the optical/NIR images during the cross-matching, the IRAC photometry is recomputed following a deconvolution method similar to that used in Grazian et al. (2006), Wuyts et al. (2008), Williams et al. (2009), or Wang et al. (2010).

In our case, first, the coordinates of the photometric aperture are re-positioned to that of the optical/NIR counterparts. Then, the PSF of the higher resolution image is convolved to the IRAC PSF, and the flux of each source is scaled to match that of the real IRAC sources measured in $00^{\prime} .9$ apertures (after recentering to the positions of the optical counterparts). Finally, total magnitudes are computed applying an aperture correction of $[1.30 \pm 0.07,1.02 \pm 0.08,1.2 \pm 0.10,1.44 \pm 0.14] \mathrm{mag}$ in the $[3.6,4.5,5.8,8.0] \mu \mathrm{m}$ bands, respectively. Figure 4 illustrates the deconvolution procedure using an HST/ACS image as reference. The red and green apertures depict the standard $2^{\prime \prime}$ aperture (for isolated sources) and the 0.9 aperture, respectively. Pixel-by-pixel variations in the residual from subtracting the model PSF do not exceed a 5\% within the 0.9 and $2^{\prime \prime}$ apertures. We also checked that the average rms $(\sim 3 \%)$ is well within the photometric error of the sources. The analysis of an average PSF, derived from observed sources across the image, indicates that for the typical separation between blended sources, $\sim 2$ ". 2 ( $>1$ 1.8 for $75 \%$ of them), the flux contamination from the nearby neighbor does not exceed a $10 \%$ for sources with a flux ratio around 1:2-3. Approximately $75 \%$ of the blended sources present flux ratios lower than 1:3.5.

After applying the deblending method, our IRAC-selected catalog contains $76,936(113,023)$ sources to [3.6] $<23.75$ (24.75). This means that we were able to deblend $8 \%$ of the sources in the original IRAC catalog built with SExtractor (presented in Section 3.1), and 16\% of the final catalog of 76,936 sources were deblended (typically, each blended sources was a combination of two sources). We find no significant difference in the brightness distribution of the blended sources compared to the resolved sources.

In the following sections, and in Paper II, we will analyze the SEDs and physical properties of the IRAC sources, concentrating in the sample with [3.6] $<23.75 \mathrm{mag}$, which count with more accurate IRAC photometry $(\mathrm{S} / \mathrm{N} \gtrsim 8)$. Therefore, this will be the working sample for the rest of the paper unless explicitly stated otherwise. Nonetheless, all the procedures
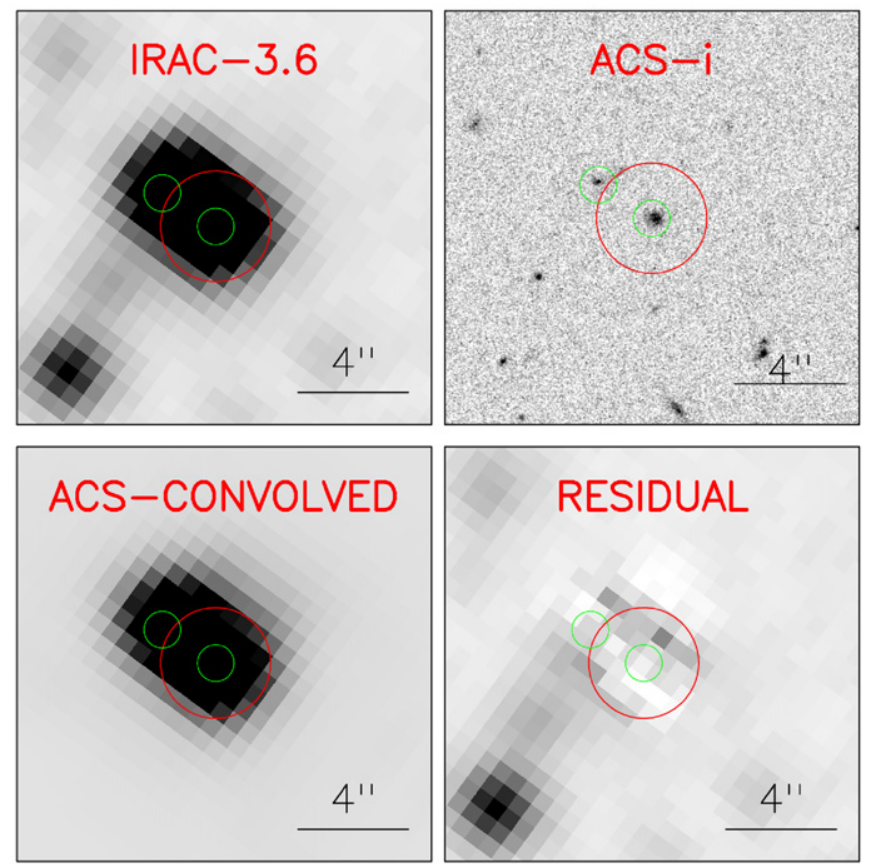

Figure 4. Example of the deconvolution procedure for unresolved sources in IRAC images. After the deblending, the unique IRAC source (irac164074) becomes two separate sources (irac164074_1 and_2). Top left (a): IRAC $3.6 \mu \mathrm{m}$ image $\left(0.0^{\prime \prime} 61\right.$ pixel $\left.^{-1}\right)$ showing a blended source. The red circle depicts a $2^{\prime \prime}$ radius aperture (our default aperture for isolated sources). The green circles show 0 ! $^{\prime} 9$ apertures centered at the positions of sources in the reference image. Top right (b): $H S T$ /ACS reference image $\left(0^{\prime} .02\right.$ pixel $\left.^{-1}\right)$ after applying a $4^{\prime} \times 4^{\prime}$ local WCS re-alignment, showing the individual sources. Bottom left (c): model of the blended source obtained by convolving the PSF of HST/ACS to the PSF of IRAC $3.6 \mu \mathrm{m}$ in (b), registering it to image (a) and scaling each source to the flux in the 0.9 apertures (green) in (a). Bottom right (d): residual from the subtraction of the model and science images $(c-a)$.

discussed in the following are also applied to the sources down to [3.6] $<24.75 \mathrm{mag}$. Although these objects are not included in the accompanying catalog (presented in Section 6, restricted to the most reliable detections), it is possible to retrieve their data through our online database (see Section 6.3).

\subsection{Merged Photometry}

The photometry is carried out in the same (Kron) elliptical aperture in all bands. The parameters of that aperture are obtained from a reference image (the same as for the crossmatching procedure) whose resolution is representative of the entire data set. Normally, this reference image is a ground-based optical/NIR frame with a PSF of approximately $1^{\prime \prime}-1$ 1.5, which is easy to translate to other ground-based images avoiding aperture issues.

The bands are sorted according to depth to facilitate the crossmatch to the optical bands. The typical aperture band for the majority of the sources (83\% of the sample) is the Subaru $R$ band, followed by the CFHTLS $i^{\prime}$ band (4\%), MMT $-i,-z$ ( $3 \%$ each) and MOIRC- $K(2 \%)$. For the remaining $5 \%$ of the sources, the aperture is computed from other bands (CFHTLS- $r$, $\left.i_{814}, V_{505}\right)$ that account for less than $1 \%$ of the total. The Subaru imaging was preferred to the CFHTLS as primary aperture band due to the uniform coverage of the whole IRAC mosaic (the CFHTLS frames cover only $\sim 60 \%$ of the IRAC survey). In order to ensure that the aperture radius is always large enough to enclose the full PSF profile in all ground-based images, we established a minimum aperture radius equal to the worst 


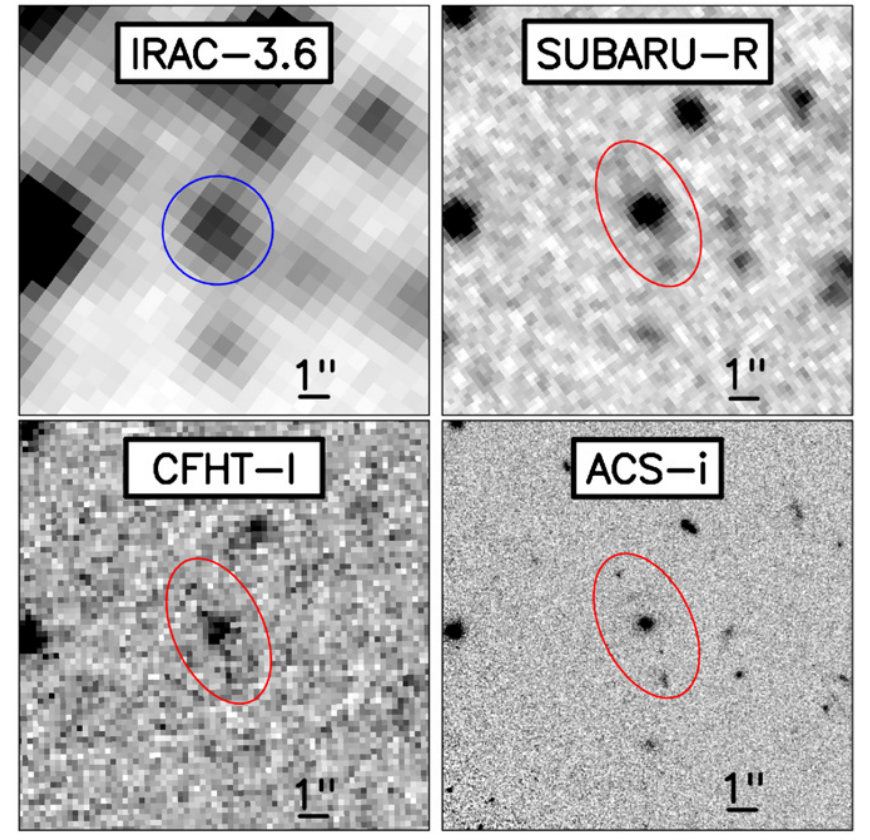

Figure 5. Multi-band images of an IRAC-selected source (irac096525) in the [3.6], $R, I$, and $i_{814}$ bands (from left to right and top to bottom). The size of the frames is $15^{\prime \prime} \times 15^{\prime \prime}$. In the three optical bands, the flux is measured in elliptical apertures (red) whose parameters are determined in the $R$-band image. Note that, despite the different image resolutions (and multiple HST counterparts), the accurate WCS re-centering ( $\left.\mathrm{rms}<00^{\prime \prime} 15\right)$ allows to place the aperture correctly, recovering the flux of all sources. For the IRAC bands, we use fixed circular apertures of $2^{\prime \prime}$ radius (blue) and we apply a correction to the total magnitude.

value of the seeing $\left(1^{\prime \prime} .5\right)$. Once the best photometric aperture is defined, if no counterpart is found in one band but data exist at that position, the flux is measured within the same aperture. Note that the local WCS re-centering of the images allows an accurate positioning of that aperture. In this way, we recover fluxes for very faint sources whose detection was missed by SExtractor. When the counter-part source is too faint to be detected, the sky-rms value is stored to be used as an upper limit in the SED analysis.

Some bands are deliberately excluded from the merged photometric measurement described above due to a significant difference of the resolution compared with the reference band or because images are not available. For all these bands, the photometry is obtained differently and later incorporated in the merged catalog during the cross-correlation procedure. For the four IRAC channels, the optical/NIR Kron apertures are typically not large enough to enclose the entire PSF. Therefore, we keep the values measured in Section 3.1 with aperture photometry. Given the comparatively large PSFs of the MIPS $(24 \mu \mathrm{m}, 70 \mu \mathrm{m})$ and GALEX (FUV, NUV) images, the flux measurement in these bands was carried with IRAFDAOPHOT and SExtractor, respectively (see PG05; PG08). Figure 5 illustrates the different resolutions and aperture sizes involved in the photometric measurement (see also the captions in Figures 9-12).

The HST -ACS bands were included in the general photometric procedure. Although the much higher resolution may lead to multiple cross identifications even when compared with the optical ground based images, the high spatial resolution (after the local re-alignment described above) allows a reliable crossmatching within 0 '.15 (rms of the local WCS solution for HST images). As shown in Figure 5, apertures were placed correctly even when multiple counterparts are identified, and the photometry includes the fluxes from all of them. We have conducted an additional test to check the accuracy of the ACS photometry measured with this method. We compared the observed photometry to synthetic magnitudes derived from SED templates fitted to the multi-band photometry (except ACS) of spectroscopically confirmed galaxies. We find a very small offset $(\leqslant 0.02 \mathrm{mag})$ and a scatter consistent with the typical photometric errors in the bands (see Section 3.1.3 of Paper II for more details).

The NIR source catalog of the Palomar/WIRC survey (Bundy et al. 2006) is also included in the merged photometric catalog, although no images are available to match apertures. In this case, we use the SExtractor MAG_AUTO value of the closest neighbor in the $J$ and $K$ bands.

\subsection{Photometric Uncertainties}

The uncertainties in the photometry are derived simultaneously to the process of flux measurement in each individual band. As mentioned in Section 3.1, the photometric errors obtained using SExtractor often underestimate the true background noise due to signal correlation on adjacent pixels (Labbé et al. 2003; Gawiser et al. 2006). In order to properly account for this effect, we estimated the flux uncertainties in three different ways, as described in PG08 (Appendix A). First, we measured the background noise in a circular corona of $5^{\prime \prime}$ width around each source. This procedure is similar to that used by SExtractor and provides uncertainties $\sigma_{\mathrm{AP}} \propto N_{\text {pixels }}^{1 / 2}$. In addition, we measured the average sky signal on non-connected artificial apertures built with random pixels around each source, with the same size as the photometric aperture, and containing only pure sky pixels (rejecting pixels $>5 \sigma$ the rms obtained with the first method). Finally, we also estimated the background noise following the method by Labbé et al. (2003). The flux measured on several photometric apertures around the source, identical to the one employed for the photometry, is fitted to a Gaussian function to yield the rms background fluctuation. The sky background is set to the resulting average value of the three methods, and the final photometric uncertainty is set to the largest estimate, typically, the one measured with the second method.

\section{PROPERTIES OF THE MERGED PHOTOMETRIC CATALOG}

The exposure time of the IRAC mosaics in the four channels is remarkably homogeneous across the mosaic. However, the coverage of the strip at other wavelengths is patchy and discontinuous (see Figure 1). For the remaining of this section and in Paper II, we will differentiate between the region of the IRAC image covered by the CFHTLS, and the rest. The CFHTLS/IRAC common region (52.16 $<\delta<53.20$ and $214.04<\alpha<215.74$ ) has been also surveyed with Subaru, the CFHT12k instrument, MMT/Megacam, HST/ACS, HST/ NICMOS, and GALEX, so it constitutes the zone with the highest data quality (hereafter main region), where the SEDs are sampled with the highest band coverage. The area of this region is $0.35 \mathrm{deg}^{2}(\sim 68 \%$ of the total) and contains 53,030 $(76,936)$ sources down to [3.6] $<23.75$ (24.75). The $0.13 \mathrm{deg}^{2}$ outside the main region (hereafter flanking regions) also present a solid SED coverage, relying mostly in the MMT and SUBARU bands. Nevertheless, it lacks some of the best-quality data (taken with ACS and MOIRCS), and the fraction of surveyed area by the WIRC- $J K$ bands is $\sim 15 \%$ lower. There are 23,906 $(35,416)$ sources in the flanking regions with [3.6] $<23.75$ 
Table 3

The IRAC 3.6+4.5 $\mu \mathrm{m}$ Sample: Detection Efficiency in Each Band and Region

\begin{tabular}{|c|c|c|c|c|c|c|c|c|c|c|c|}
\hline \multirow{3}{*}{$\begin{array}{l}\text { Band } \\
\text { (1) }\end{array}$} & \multirow{3}{*}{$\begin{array}{l}\lambda_{\text {eff }} \\
\\
\text { (2) }\end{array}$} & \multicolumn{5}{|c|}{ Main Region $\left(0.35 \mathrm{deg}^{2}\right)$} & \multirow{3}{*}{$\begin{array}{c}\text { Area } \\
\text { (8) }\end{array}$} & \multicolumn{4}{|c|}{ Flanking Regions $\left(0.13 \mathrm{deg}^{2}\right)$} \\
\hline & & \multirow{2}{*}{$\begin{array}{l}\text { Area } \\
\text { (3) }\end{array}$} & \multicolumn{2}{|c|}{$[3.6]<23.75[53,030]$} & \multicolumn{2}{|c|}{$[3.6]<24.75[77,607]$} & & \multicolumn{2}{|c|}{$[3.6]<23.75[23,906]$} & \multicolumn{2}{|c|}{$[3.6]<24.75[35,416]$} \\
\hline & & & $\begin{array}{c}\text { Stats } \\
(4)\end{array}$ & $\begin{array}{c}\mathrm{All} / \mathrm{S} / \mathrm{N}>5(\%) \\
(5)\end{array}$ & $\begin{array}{l}\text { Stats } \\
(6)\end{array}$ & $\begin{array}{c}\text { All } / \mathrm{S} / \mathrm{N}>5(\%) \\
(7)\end{array}$ & & $\begin{array}{l}\text { Stats } \\
(9)\end{array}$ & $\begin{array}{c}\text { All } / \mathrm{S} / \mathrm{N}>5(\%) \\
(10)\end{array}$ & $\begin{array}{l}\text { Stats } \\
(11)\end{array}$ & $\begin{array}{c}\text { All } / \mathrm{S} / \mathrm{N}>5(\%) \\
(12)\end{array}$ \\
\hline X-ray ${ }^{a}$ & $0.31,1.24 \mathrm{~nm}$ & 0.97 & $\ldots$ & $717(1.3 \%)$ & & $718(1.3 \%)$ & 0.93 & & $307(1.2 \%)$ & $\ldots$ & $307(1.2 \%)$ \\
\hline FUV & 0.154 & 0.99 & $27.90_{25.78}^{29.45}$ & $58.8 / 7.9$ & $28.03_{26.19}^{29.55}$ & $57.4 / 6.1$ & 0.15 & $27.71_{25.94}^{29.27}$ & $59.6 / 7.7$ & $27.83_{26.27}^{29.34}$ & $58.3 / 6.8$ \\
\hline NUV & 0.232 & 0.99 & $26.28_{24.47}^{28.16}$ & $74.2 / 25.8$ & $26.60_{24.78}^{28.35}$ & $70.8 / 20.2$ & 0.15 & $26.26_{24.44}^{28.11}$ & $75.2 / 26.7$ & $26.57_{24.81}^{28.33}$ & $72.5 / 23.7$ \\
\hline$u^{\prime}$ & 0.363 & 1.00 & $25.53_{24.29}^{27.01}$ & $88.4 / 52.8$ & $25.85_{24.61}^{27.25}$ & $86.1 / 44.1$ & 1.00 & $25.39_{24.15}^{26.78}$ & $89.6 / 64.3$ & $25.69_{24.45}^{27.03}$ & $88.0 / 57.8$ \\
\hline$u^{*}$ & 0.381 & 1.00 & $25.30_{24.13}^{26.68}$ & $88.2 / 53.9$ & $25.60_{24.43}^{26.93}$ & $85.2 / 44.2$ & $\ldots$ & $\ldots$ & $\ldots$ & $\ldots$ & $\ldots$ \\
\hline CFH- $B$ & 0.440 & 1.00 & $25.03_{23.82}^{26.26}$ & $89.6 / 55.2$ & $25.33_{24.16}^{26.56}$ & $87.1 / 45.6$ & 1.00 & $24.94_{23.73}^{26.20}$ & $87.3 / 53.1$ & $25.27_{24.05}^{26.48}$ & $83.7 / 43.0$ \\
\hline$g$ & 0.481 & 1.00 & $25.08_{23.85}^{26.31}$ & $96.1 / 85.1$ & $25.44_{24.23}^{26.64}$ & $95.0 / 80.3$ & 1.00 & $25.12_{23.76}^{26.40}$ & $96.6 / 84.9$ & $25.49_{24.14}^{26.71}$ & $95.5 / 80.5$ \\
\hline$g^{\prime}$ & 0.486 & 1.00 & $24.86_{23.65}^{26.05}$ & $95.4 / 83.6$ & $25.20_{24.01}^{26.34}$ & $94.0 / 78.2$ & $\ldots$ & $\ldots$ & $\ldots$ & $\ldots$ & $\ldots$ \\
\hline$V_{606}$ & 0.592 & 0.56 & $24.58_{23.23}^{25.71}$ & $94.7 / 83.5$ & $24.92_{23.66}^{26.00}$ & $93.9 / 78.7$ & $\ldots$ & $\ldots$ & $\ldots$ & $\ldots$ & $\ldots$ \\
\hline$r^{\prime}$ & 0.626 & 1.00 & $24.50_{23.08}^{25.56}$ & $97.1 / 89.3$ & $24.85_{23.52}^{25.93}$ & $96.0 / 84.2$ & $\ldots$ & $\ldots$ & $\ldots$ & $\ldots$ & $\ldots$ \\
\hline$R$ & 0.652 & 1.00 & $24.44_{23.00}^{25.45}$ & $97.0 / 88.5$ & $24.80_{23.44}^{25.82}$ & $95.8 / 82.5$ & 1.00 & $24.36_{22.81}^{25.44}$ & $97.4 / 87.7$ & $24.74_{23.27}^{25.80}$ & $96.5 / 81.9$ \\
\hline CFH- $R$ & 0.660 & 1.00 & $24.49_{22.98}^{25.58}$ & $95.3 / 71.5$ & $24.85_{23.45}^{25.96}$ & $93.1 / 60.2$ & 1.00 & $24.36_{22.73}^{25.50}$ & $91.6 / 73.7$ & $24.76_{23.22}^{25.90}$ & $88.4 / 63.0$ \\
\hline$i^{\prime}$ & 0.769 & 1.00 & $24.00_{22.43}^{25.14}$ & $95.3 / 85.4$ & $24.41_{22.92}^{25.52}$ & $94.2 / 78.3$ & $\ldots$ & $\ldots$ & $\ldots$ & $\ldots$ & $\ldots$ \\
\hline$i$ & 0.782 & 1.00 & $24.09_{22.52}^{25.15}$ & $97.4 / 90.3$ & $24.51_{23.01}^{25.62}$ & $96.1 / 83.8$ & 1.00 & $24.13_{22.25}^{25.31}$ & $96.4 / 81.1$ & $24.54_{22.80}^{25.87}$ & $94.6 / 70.2$ \\
\hline$i_{814}$ & 0.807 & 0.56 & $24.11_{22.49}^{25.23}$ & $96.7 / 80.7$ & $24.52_{23.00}^{25.68}$ & $94.7 / 69.3$ & $\ldots$ & $\ldots$ & $\ldots$ & $\ldots$ & $\ldots$ \\
\hline CFH-I & 0.813 & 1.00 & $24.03_{22.34}^{25.23}$ & $94.1 / 68.6$ & $24.45_{22.83}^{25.70}$ & $90.1 / 54.5$ & 1.00 & $23.97_{22.07}^{25.25}$ & $89.2 / 61.1$ & $24.43_{22.58}^{25.70}$ & $84.1 / 47.5$ \\
\hline$z^{\prime}$ & 0.887 & 1.00 & $23.79_{22.17}^{24.98}$ & $93.3 / 66.2$ & $24.23_{22.61}^{25.48}$ & $89.1 / 51.2$ & $\ldots$ & $\ldots$ & $\ldots$ & $\ldots$ & $\ldots$ \\
\hline$z$ & 0.907 & 1.00 & $23.96_{22.30}^{25.18}$ & $96.0 / 77.7$ & $24.44_{22.75}^{25.66}$ & $92.7 / 64.9$ & 1.00 & $23.95_{22.15}^{25.25}$ & $97.5 / 85.5$ & $24.44_{22.65}^{25.73}$ & $95.3 / 75.4$ \\
\hline$J_{110}$ & 1.10 & 0.04 & $23.87_{22.38}^{24.94}$ & $84.6 / 65.4$ & $24.25_{22.79}^{25.48}$ & $88.9 / 55.6$ & $\ldots$ & $\ldots$ & $\ldots$ & $\ldots$ & $\ldots$ \\
\hline$\Omega 2 k-J$ & 1.21 & 0.40 & $23.29_{21.81}^{24.50}$ & $93.7 / 38.5$ & $23.74_{22.21}^{25.01}$ & $88.4 / 27.1$ & $\ldots$ & $\ldots$ & $\ldots$ & $\ldots$ & $\ldots$ \\
\hline WIRC-J & 1.24 & 0.42 & $22.43_{20.90}^{23.47}$ & $62.8 / 16.6$ & $22.49_{20.96}^{23.59}$ & $46.1 / 11.7$ & 0.32 & $22.31_{20.69}^{23.41}$ & $57.9 / 20.4$ & $22.35_{20.73}^{23.46}$ & $41.1 / 14.1$ \\
\hline$H_{160}$ & 1.59 & 0.04 & $24.71_{23.19}^{25.84}$ & $92.3 / 80.8$ & $25.18_{23.61}^{26.52}$ & $91.7 / 69.4$ & $\ldots$ & $\ldots$ & $\ldots$ & $\ldots$ & $\ldots$ \\
\hline$\Omega^{\prime}-K_{s}$ & 2.11 & 0.46 & $22.43_{20.89}^{23.73}$ & $75.8 / 6.7$ & $22.74_{21.38}^{23.98}$ & $67.0 / 4.6$ & 0.26 & $22.17_{20.72}^{23.46}$ & $67.3 / 7.3$ & $22.39_{21.04}^{23.76}$ & $59.6 / 5.0$ \\
\hline$K s$ & 2.15 & 0.26 & $24.64_{23.03}^{25.77}$ & $95.5 / 73.8$ & $25.14_{23.44}^{26.42}$ & $89.8 / 52.9$ & $\ldots$ & $\ldots$ & $\ldots$ & $\ldots$ & $\ldots$ \\
\hline WIRC- $K$ & 2.17 & 0.97 & $21.76_{20.21}^{22.74}$ & $62.6 / 27.1$ & $21.82_{20.26}^{22.81}$ & $42.6 / 16.3$ & 0.80 & $21.42_{19.86}^{22.50}$ & $45.3 / 18.4$ & $21.45_{19.89}^{22.55}$ & $42.4 / 14.4$ \\
\hline IRAC 36 & 3.56 & 1.00 & $22.54_{20.97}^{23.41}$ & $100.0 / 99.2$ & $23.18_{21.43}^{24.25}$ & $100.0 / 84.5$ & 1.00 & $22.52_{20.87}^{23.40}$ & $100.0 / 97.8$ & $23.17_{21.39}^{24.24}$ & $100.0 / 80.6$ \\
\hline IRAC 45 & 4.51 & 1.00 & $22.62_{21.05}^{23.55}$ & $99.8 / 95.3$ & $23.26_{21.51}^{24.44}$ & $98.9 / 75.3$ & 1.00 & $22.63_{21.00}^{23.61}$ & $99.3 / 91.1$ & $23.27_{21.44}^{24.49}$ & $97.1 / 70.5$ \\
\hline IRAC 58 & 5.69 & 1.00 & $22.50_{21.04}^{23.73}$ & $89.7 / 41.1$ & $22.89_{21.33}^{24.28}$ & $78.4 / 28.0$ & 1.00 & $22.45_{20.95}^{23.71}$ & $87.1 / 38.6$ & $22.80_{21.22}^{24.20}$ & $75.5 / 26.4$ \\
\hline IRAC 80 & 7.96 & 1.00 & $22.62_{21.14}^{23.89}$ & $85.8 / 34.4$ & $22.97_{21.37}^{24.33}$ & $73.8 / 23.5$ & 1.00 & $22.51_{21.05}^{23.83}$ & $82.6 / 32.9$ & $22.83_{21.29}^{24.26}$ & $71.1 / 22.6$ \\
\hline MIPS 24 & 23.84 & 0.91 & $19.58_{18.44}^{20.59}$ & $31.5 / 20.2$ & $19.71_{18.50}^{20.72}$ & $29.7 / 18.1$ & 0.93 & $19.36_{18.35}^{20.39}$ & $29.9 / 19.1$ & $19.49_{18.42}^{20.53}$ & $25.2 / 15.1$ \\
\hline MIPS 70 & 72.49 & 0.80 & $15.79_{15.23}^{16.37}$ & $13.1 / 1.5$ & $15.81_{15.25}^{16.35}$ & $7.5 / 5.7$ & 0.86 & $15.57_{14.86}^{16.24}$ & $13.6 / 1.2$ & $15.57_{14.87}^{16.24}$ & $6.7 / 4.7$ \\
\hline \multirow[t]{2}{*}{$\mathrm{VLA}^{\mathrm{a}}$} & $2 \mathrm{E} 06$ & 0.74 & $\ldots$ & $380(<1 \%)$ & $\ldots$ & $380(<1 \%)$ & 0.42 & $\ldots$ & $210(<1 \%)$ & $\ldots$ & $210(<1 \%)$ \\
\hline & & & \multicolumn{2}{|c|}{$[3.6]<23.75[53,030]$} & \multicolumn{2}{|c|}{$[3.6]<24.75[77,607]$} & & \multicolumn{2}{|c|}{$[3.6]<23.75[23,906]$} & \multicolumn{2}{|c|}{$[3.6]<24.75[35,416]$} \\
\hline Redshifts $^{\mathrm{a}}$ & $0.64-0.91$ & 0.94 & 6191 & $12.0 \%$ & 6420 & $8.4 \%$ & 0.84 & 1445 & $6.0 \%$ & 1481 & $4.2 \%$ \\
\hline
\end{tabular}

Notes. Detection efficiency for the sources IRAC $3.6+4.5 \mu \mathrm{m}$ sample in the different bands compiled for this work. The analysis is divided in two zones: the main region, defined as the overlapping area between the IRAC and the CFHTLS mosaics (52.16 $<\delta<53.20$ and $\alpha>214.04)$, and the flanking region. (1) Band name. (2) Effective wavelength of the bands in microns. (3) Fraction of the main region covered by the observations in each band. $(4,6)$ Median and quartiles of the magnitude distribution in each band for main region sources with [3.6] $<23.75$ and [3.6] $<24.75$, respectively. $(5,7)$ Percentage of IRAC sources in the main region detected in each band at any magnitude (All) and with $\mathrm{S} / \mathrm{N}>5$. These values are computed in areas fully covered by both the IRAC mosaic and the observations in each band. (8) Same as $(3)$ in the flanking regions. $(9,11)$ Same as $(4,6)$ in the flanking regions. $(10,12)$ Same as $(5,7)$ in the flanking regions.

${ }^{a}$ For these catalogs, we quote only the number of sources detected in IRAC, and the percentage of total IRAC sample that they represent.

(24.75). Table 2 summarizes the number of sources in the catalog according to different limiting magnitudes and area constraints.

Note that the bulk of the optical SED coverage is based on CFHTLS and MMT data (the CFHT12k-BRI data are $\sim 2$ mag shallower). On average numbers, their respective filter sets cover a similar wavelength range with comparable data quality (see Tables 1 and 3). Also, the subtle differences in the shape of the $u$ - and $z$-band filters of each survey improve the quality of the SED coverage for the sources in the main region. The most remarkable difference between the two surveys is found in the homogeneity of data; whereas the CFHTLS presents a nearly uniform data quality across the mosaic, the MMT data show larger variations between the four pointings (e.g., $\Delta g(5 \sigma)=$ 26-25 mag, $\Delta \mathrm{FWHM}=1$ ".0-1".6; see Zhao et al. 2009 for more details). Unfortunately, the lowest quality pointings (shallower data and highest seeing) are precisely those covering the flanking regions (mainly the north region, $\delta>53.20$ ). Furthermore, a small area in the north and south regions of the MMT mosaic 
$(<10 \%$ of the total) is not observed in the $g$ and $u$ bands, respectively. Thus, comparatively, the average data quality in the flanking regions is slightly lower than in the main region.

\subsection{Multi-band Detection and Color Properties}

Table 3 shows the fraction of the entire IRAC catalog detected in the different bands compiled for this work. We give specific values for different sub-samples divided as a function of brightness in the selection band, detection level $(\mathrm{S} / \mathrm{N})$, and location in the IRAC mosaic. The majority of sources are detected in the four IRAC channels. However, while most sources $(>90 \%)$ have reliable detections at 3.6 and $4.5 \mu \mathrm{m}$, only half of the sample is detected with $\mathrm{S} / \mathrm{N}>5$ at the two longer wavelengths. Note that the photometry at 5.8 and $8.0 \mu \mathrm{m}$ was not performed independently (see Section 3.1).

The fraction of IRAC sources detected in the optical bands is also elevated ( $\sim 95 \%)$ for the majority of the bands. However, cutting the measurements at $\mathrm{S} / \mathrm{N}>5$, the efficiency decreases to $\sim 85 \%$ for the deepest CFHTLS, MMT, Subaru and ACS bands, and to $50 \%-60 \%$ for the $u$ and $z$ bands (in both CFHTLS and MMT data) and the shallower CFHT BRI bands. For the GALEX data, the detection is typically lower than $10 \%$ and $25 \%$ in the FUV and NUV bands, respectively. Around $12 \%$ and $6 \%$ of the sources are matched to high-quality spectroscopic redshift estimates in the main and flanking regions, respectively. The lower efficiency in the flanking regions is caused by the inhomogeneous DEEP2 coverage of the IRAC mosaic.

The NIR coverage of the strip surveyed by IRAC is also discontinuous. The WIRC catalog provides the most uniform coverage, including $40 \%$ and $\sim 100 \%$ of the area in the main region in the $J$ and $K$ bands, respectively. The fraction of IRAC sources detected in each band is very similar, $\sim 50 \%-60 \%$ ( $\sim 20 \%$ with $\mathrm{S} / \mathrm{N}>5$ ). The deepest NIR observations are those taken in CAHA- $J$ and Subaru-MOIRCS- $K_{s}$ bands. The latter covers $\sim 1 / 4$ of the main region up to $1 \mathrm{mag}$ deeper than the WIRC- $K$ data, presenting a much higher detection fraction, $\sim 90 \%(70 \%$ with $\mathrm{S} / \mathrm{N}>5)$. The CAHA$J$ data cover $40 \%$ of the main region recovering $\sim 30 \%(\mathrm{~S} /$ $\mathrm{N}>5$ ) of the IRAC detections, a slightly larger fraction than WIRC-J.

We note that for most bands, the fraction of sources detected with $\mathrm{S} / \mathrm{N}<5$ can be significantly higher than the overall value. In the shallowest bands, this is caused by the higher photometric uncertainties (e.g., the WIRC $J$ and $K$ bands). However, for most bands this is the result of the enhanced detection achieved with the forced photometric measurement. For each IRAC source undetected in a given image, but having a counterpart in any other band, we still measure the flux in the same aperture at the position of the counterpart. With this method, we increase the detection efficiency at fainter magnitudes recovering sources that would be missed otherwise. However, given the extreme faintness of these sources, some of the measurements produce low significance detections $(\mathrm{S} / \mathrm{N}<2)$. As a precaution, to preserve the overall quality of the SED, we do not include these values (photometric uncertainties $>0.4 \mathrm{mag}$ ) in the SED fitting procedure, nor in the estimate of the physical parameters in Paper II. Nevertheless, we keep these values as they can be useful as upper limits. Another estimate of the limiting magnitude in each band is obtained from the value of the sky rms in failed forced measurements (i.e., those for which the measured flux in the aperture is negative).

Figure 6 illustrates the characteristics of the different flux measurements in four bands probing representative wavelengths in the UV-to-NIR range. The central panel of each plot shows a color-magnitude diagram in [3.6] versus $u^{*} R i^{\prime}$ and $K$, respectively. The gray-scale density map depicts the distribution of standard (non-forced) photometric measurements, typically detected with $\mathrm{S} / \mathrm{N}>5$ (magenta line). Forced detections for which we obtain a valid flux or a sky-rms value are shown as green and blue dots, respectively. The cyan markers are sources detected in IRAC only (for which we assign arbitrary magnitudes in each band). For the deep $R$ and $i^{\prime}$ bands, which present the highest detection efficiency, forced measurements account for less than $5 \%$ of the flux measurements and present a median $\mathrm{S} / \mathrm{N} \lesssim 2$ (black line). In the $u^{*}$ band, the source density for a similar limiting magnitude is much lower, and consequently the fraction of forced detections is higher, around $20 \%$.

The upper panels in each quadrant of Figure 6 show the fraction of undetected sources in each band. In the $R$ and $i^{\prime}$ bands, these sources make up for $<4 \%$ of the total sample. Most of them are IRAC-only sources (plotted in cyan), whereas the rest are at least marginally detected in one other (typically red) band, but the forced measurement fails for the particular band shown in the plot (blue). The fraction of undetected sources in the $u^{*}$ band (and other shallower optical bands, e.g., CHFT12K BRI) increases to $10 \%$, being in this case dominated by failed forced measurements at the positions of $R$ or $i^{\prime}$ detections. These kinds of measurement are also predominant for undetected sources in the $K$ band.

Note that some of the IRAC-only sources are relatively bright, $21<[3.6]<22$. Most of them are detected in deep $K$-band observations. However, there are a few galaxies detected only in the IRAC bands (typically the faintest ones). The nature of these interesting sources, potential candidates to massive highredshift galaxies, will be explored in a future paper.

Figure 6 also shows the color distribution of IRAC sources in bins of [3.6] mag (right panels in each quadrant) and spectroscopically confirmed galaxies in several redshift bins (red ellipses and dots in the central panels). The general trend with color in the $R$ and $i^{\prime}$ bands is that faint IRAC sources are on average bluer than brighter ones. The median colors for the faintest [3.6] bin, $22.50<[3.6]<23.75$, are $R-[3.6]=1.6$ and $i^{\prime}-[3.6]=1.4$. These colors are similar to those of galaxies at $1<z<1.5$. This is consistent with the fact that the median redshift for the IRAC $3.6+4.5 \mu \mathrm{m}$ magnitude limited sample $([3.6]<23.75)$ is $z \sim 1$ (see Paper II and PG08). Note also that most of the forced detections in the $R$ band would qualify as IRAC extremely red objects (by the criteria of Yan et al. 2004, $R-[3.6]>4.0)$ that target dusty starbursts or passively evolving galaxies at $z>1.5$.

In general, all galaxies tend to become redder in optical-IRAC colors with increasing redshifts. Indeed, for a typical galaxy SED, the observed optical bands shift into the (fainter) UV whereas the [3.6] mag becomes brighter as it approaches the stellar bump at $1.6 \mu \mathrm{m}$ rest frame (for $z \lesssim 1.2$ ). Therefore, it is not surprising that the $u^{*}-[3.6]$ median color is redder $\left(u^{*}-[3.6]=2.1\right.$, typical of a $z \sim 1$ galaxy) than the median color involving the $\mathrm{Ri}^{\prime} \mathrm{K}$ bands. Note also that the observed UV progressively shifts into the Lyman break, producing the large fraction of $z>2.5$ (red dots in the top left quadrant of Figure 6) galaxies that are $u$-dropouts. Finally, we find that the $K-$ [3.6] color presents an almost constant value as a function of [3.6] mag for a given redshift range, becoming progressively redder as we move to higher redshifts: $K-[3.6]<0$ at $z<0.5$ and $K-[3.6]>0$ at $z>1$. Again, this is 

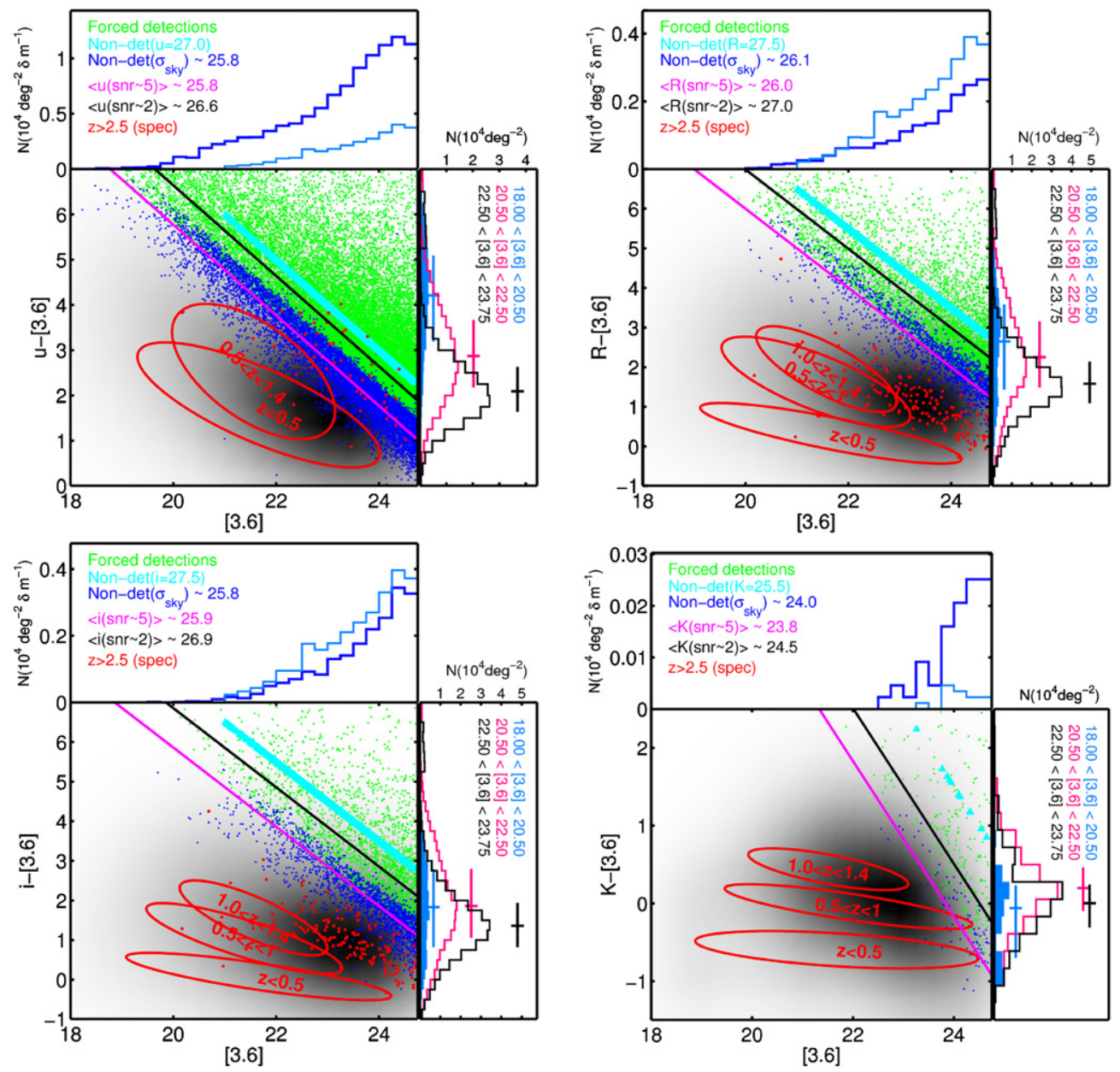

Figure 6. Central panel in each plot shows the color magnitude diagrams in the $u^{*}, R, i^{\prime}$, and $K$ bands with respect to [3.6] (top to bottom, left to right). The density map in gray scale shows the distribution of IRAC sources detected in each band. For sources missing (not detected by SExtractor) in a given band but detected in any other, we force a flux measurement at the position of the existing source using the same Kron aperture. The green dots depict sources for which we are able to recover a positive flux in this forced measurement. The blue dots depict the value of the sky rms in the apertures where the forced measurement failed (i.e., the integrated flux was negative). The cyan triangles are sources undetected in any other band but IRAC. For these sources, we set an upper limiting magnitude $\sim 0.5-1$ mag fainter than the typical magnitude for a source with $\mathrm{S} / \mathrm{N}=2$ (black line). The magenta solid line indicates the median magnitude of the sources with $\mathrm{S} / \mathrm{N} \sim 5$. The red dots depict galaxies with spectroscopic redshift $z>2.5$. The red ellipses show the median and quartiles of the color-magnitude distribution for galaxies in different bins of redshift within $0<z<1.5$. The upper panel of each quadrant shows the [3.6] brightness distribution of undetected sources (blue and cyan markers in the central panel) in each band. The right panel of each quadrant shows the color distribution of detected sources (histogram, median, and quartiles) in three bins of the [3.6] magnitude.

consistent with the fact that both $K$ and [3.6] transit through the peak of the stellar bump for $z<1$, changing their relative positions (see, e.g., Huang et al. 2004). An interesting consequence of the red $K-$ [3.6] colors of high- $z$ galaxies is that IRAC $3.6 \mu \mathrm{m}$ observations are equivalent, in terms of source densities, to a $K$-selected sample down to slightly deeper limiting magnitudes.

Figure 7 shows the density map (central panel) and histograms (right panel) of the number of photometric bands with measured fluxes, $N$ (band), in the main (left) and flanking regions (right). Typically, the SED of a galaxy in the main region has a median of 19 photometric data points. The average spectral coverage is $\sim 8$ bands larger than in the flanking regions, mainly due to the lack of data from the CFHTLS and NIR surveys. Galaxies with an available spectroscopic redshift $(R \lesssim 24 \mathrm{mag}$; green dots) typically present the highest band coverage ( $\sim 22$ and $\sim 14$ bands in the main and flanking regions, respectively), since they are relatively bright in the optical. Interestingly, the faintest IRAC sources, [3.6] > $23.75 \mathrm{mag}$ (red histogram), present a relatively high band coverage, $N($ band $)=17$ in the main region.
In most of the cases, these are low significance detections in the deepest optical/NIR bands where the forced photometric measurement (see Section 4.2) is able to recover a flux (see, e.g., Figure 9). On the opposite side, there is a non-negligible number of IRAC-faint but optically bright sources with more than 19 photometric data points. These constitute a population of blue dwarf galaxies at intermediate redshift easily detected in the optical but with faint IRAC counterparts (i.e., not very massive).

The upper panel of Figure 7 shows the [3.6] magnitude distribution for the full sample (black) and for sources with poor spectral coverage $(N$ (band) $<5$; blue histogram). The latter defines a clearly isolated group in the density contours (central panel) and in the histograms (right panel) of Figure 7. These sources represent less than $3 \%(4 \%)$ of the sample up to [3.6] $<23.75$ (24.75). Most of them are clear IRAC-only detections (as the ones discussed in Figure 6) relatively bright in [3.6,4.5]. However, some of the faintest sources can be affected by contamination of spurious sources (we give more details on these sources in Section 5.3). 

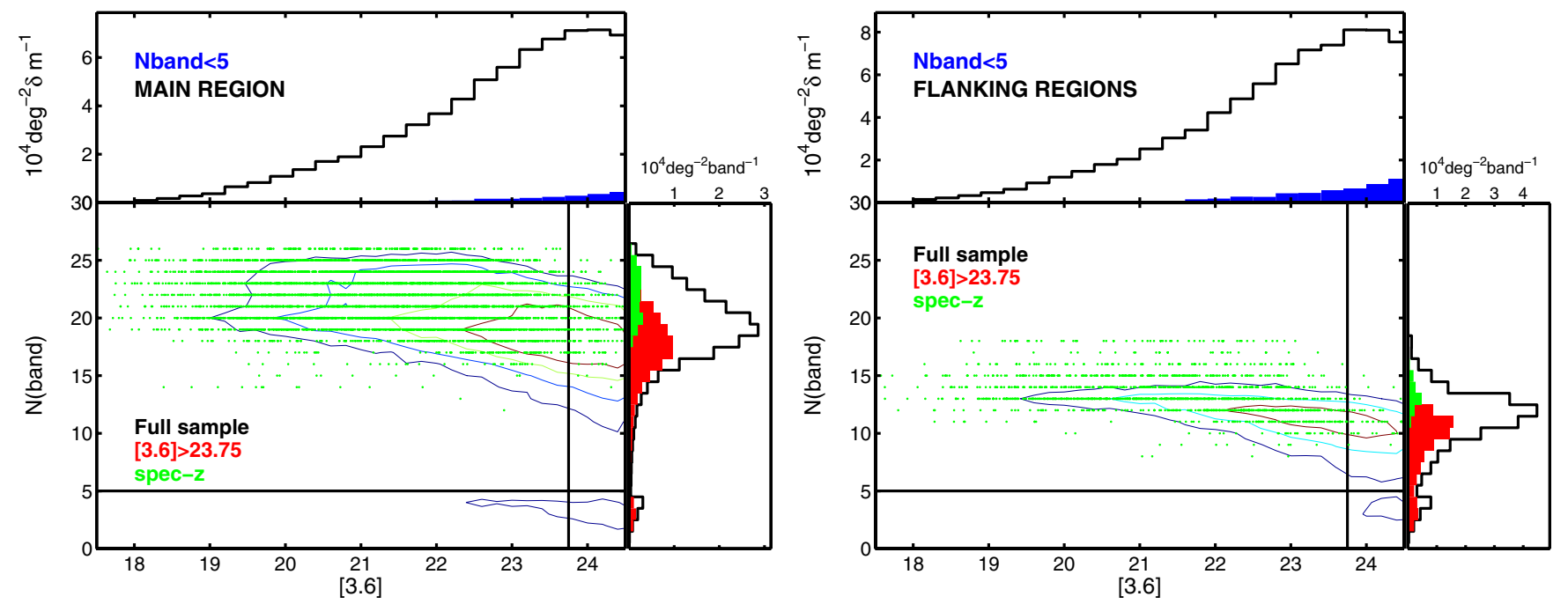

Figure 7. Central panel in each plot depicts the source density as function of the number of bands in which the source was detected (a positive flux was measured), $N$ (band), and the magnitude in the [3.6] channel, for the main region (left) and the flanking regions (right). The color contours contain (from the inside out) approximately $25 \%, 50 \%, 75 \%$, and $90 \%$ of the sources. The green dots mark galaxies with a spectroscopic redshift. The black lines indicate the $85 \%$ completeness limit of the catalog ([3.6] $<23.75$, vertical) and the band coverage limit $N$ (band) $=5$ (horizontal), respectively. The histograms on top show the brightness distribution for the full sample (black) and for sources with $N$ (band) $<5$ (blue). The histograms to the right show the $N$ (band) distribution of the full sample (black line), IRAC faint sources ([3.6] > 23.75; red area) and galaxies with spectroscopic redshifts (green area).

\subsection{FIR, X-Ray, and Radio Counterparts}

Table 3 shows the fraction of IRAC sources in our sample ([3.6] < 23.75) detected in the X-ray, FIR, and radio surveys within the overlapping area. About $30 \%$ (20\% with S/N > 5 detections) of the IRAC sources are detected at $24 \mu \mathrm{m}$ $(\mathrm{S} / \mathrm{N}=5$ reached at $\sim 60 \mu \mathrm{Jy}), 10 \%(2 \%$ with $\mathrm{S} / \mathrm{N}>5)$ at $70 \mu \mathrm{m}(\mathrm{S} / \mathrm{N}=5$ reached at $\sim 3.5 \mathrm{mJy}), 1 \%$ in the X-rays, and $\leqslant 1 \%$ at $20 \mathrm{~cm}$. For the radio and X-ray surveys, we crossmatched our sample to the catalogs of Ivison et al. (2007) and Laird et al. (2009), respectively. As explained in Section 4, the cross-match to the radio catalog was performed using a $3^{\prime \prime}$ radius, whereas for the X-ray catalog we followed a twostep identification. First, we divided the X-ray sample attending to the availability of pre-identified IRAC counterparts (Laird et al. used the IRAC catalog of BAR08 as reference). For the galaxies identified in IRAC, we used a 1" cross-matching radius, whereas for the other we used a more conservative $2^{\prime \prime}$ radius. With this procedure, we found 848 out of the 882 galaxies with IRAC counterparts in their catalog (815 among the 830 with high reliability flag; see Laird et al. 2009 for more details). The remaining galaxies were either outside of the $N$ (frame) $>20$ region or too close to a bright star. In addition, we were able to identify 175 additional $\mathrm{X}$-ray sources in the area of our mosaic not covered by the data of BAR08, i.e., we recover a total of 1023 out of $1325 \mathrm{X}$-ray sources from the catalog of Laird et al. For the MIPS $24 \mu \mathrm{m}$ and $70 \mu \mathrm{m}$ and radio surveys, we identify 10758 , 868 , and 590 (out of 1122) sources, respectively. Noticeably, about $20 \%$ and $54 \%$ of the sources detected in MIPS $24 \mu \mathrm{m}$ and $70 \mu \mathrm{m}$ present a multiple IRAC counterparts (typically $2-3$ ) and $\sim 6 \%$ in X-ray and Radio.

All of these surveys cover an area larger than the IRAC observations, but none of them fully cover the IRAC strip (although the X-ray and MIPS $24 \mu \mathrm{m}$ data cover $>90 \%$ of the area). Table 4 shows the total area covered by the X-ray, MIPS, and radio surveys, the fraction that area in common with the IRAC mosaic, and the number of sources with an IRAC counterpart in our catalog. All the sources in the different surveys located within the area covered by IRAC are detected
Table 4

Detection Efficiency of X-ray, FIR, and Radio Sources

\begin{tabular}{lccc}
\hline \hline $\begin{array}{l}\text { Source } \\
(1)\end{array}$ & $\begin{array}{c}\text { Area } \\
(2)\end{array}$ & $\begin{array}{c}\text { Fraction } \\
(3)\end{array}$ & $\begin{array}{c}\text { Total } \\
(4)\end{array}$ \\
\hline Chandra $/$ ACIS $^{\mathrm{a}}$ & $0.67 \mathrm{deg}^{2}$ & 0.70 & $1023(77 \%)$ \\
MIPS $24 \mu \mathrm{m}(f>60 \mu \mathrm{Jy})^{\mathrm{b}}$ & $0.53 \mathrm{deg}^{2}$ & 0.59 & 10771 \\
MIPS $70 \mu \mathrm{m}(f>3500 \mu \mathrm{Jy})^{\mathrm{b}}$ & $0.50 \mathrm{deg}^{2}$ & 0.61 & 868 \\
VLA $20 \mathrm{~cm}^{\mathrm{c}}$ & $0.73 \mathrm{deg}^{2}$ & 0.46 & $590(52 \%)$ \\
\hline
\end{tabular}

Notes. (1) Name of the band. (2) Total area of the survey. (3) Fraction of the survey area overlapping with the IRAC mosaic. (4) Number of sources with IRAC counterparts $([3.6]<23.75)$ and fraction of recovered sources from the whole catalog.

a Sources drawn from Laird et al. (2009). These include 815 high reliability identifications (based on a previous match to the IRAC catalog of BAR08) and 175 previously unidentified sources.

b The area of the survey refers to the GTO+FIDEL observations reduced for this paper.

${ }^{c}$ Sources drawn from Ivison et al. (2007).

in our catalog (with the exception of a few objects too close to bright stars).

Note that despite the larger area of the VLA observations, this survey covers only $\sim 40 \%$ of the region surveyed by IRAC. In fact the data are limited only to the upper region of the IRAC mosaic $(\delta>53.10$; see Figure 1$)$.

\subsection{Catalog Reliability}

In this section, we analyze in more detail the sources with a poor spectral coverage to asses the reliability of the IRAC catalog as a function of the magnitude in the [3.6] band.

First, we test the reliability with the widely used method of comparing the number of detections at faint levels in the original and a negative image. This test reveals that only $\sim 1 \%$ of the sources up to [3.6] $<23.75 \mathrm{mag}$ are spurious.

However, this procedure is conceived to detect faint spurious sources based on the assumption of a symmetric noise, whereas most of the spurious detections are concentrated around bright regions as a consequence of PSF and saturation artifacts and an 
excessive deblending. Therefore, we chose to follow a different approach to analyze the reliability of the catalog based on the fact that faint $[3.6,4.5]$ detections lacking a measurable counterpart in other bands are potential candidates for spurious detections.

As discussed in Section 5, we find a nearly isolated group of sources detected in $N$ (band) $\leqslant 4$. Most of these sources are IRAC-only detections (cyan histogram of Figure 6) that, in the absence of low significance optical detections to validate them, could be artifacts of the source extraction procedure.

The visual inspection of galaxies with $N$ (band $) \leqslant 4$ reveals a clear dichotomy. We find that these sources are either strongly clustered around bright stars and extended sources (typically low- $z$ galaxies), or uniformly distributed across the image. Most of the sources in the first group are easily identified as spurious, whereas for the isolated sources, their high $\mathrm{S} / \mathrm{N}(\sim 5-10)$ and the visual inspection seem to favor that they are real. We dealt with the reliability of sources with $N$ (band) $<4$ by considering spurious all detections in a $20^{\prime \prime}-30^{\prime \prime}$ radius region around the brightest objects $([3.6]=16-15 \mathrm{mag})$. Up to [3.6] $\leqslant 23.75$, approximately half of the $N$ (band) $\leqslant 4$ sources are spurious by this criteria, accounting for less than $2 \%$ of the total sample. Nevertheless, even for the more conservative scenario, the sum of all $N$ (band) $\leqslant 4$ detections constitutes less than $\sim 4 \%$ of the sample in the main region. Finally, we also check the reliability of these sources by studying how many of them are simultaneously detected at both 3.6 and $4.5 \mu \mathrm{m}$. Unfortunately, we find that some real detections are missed at $4.5 \mu \mathrm{m}$ due to the slightly worse image quality, while some of the spurious sources are detected in both images in regions where the density of artifacts is larger (i.e., around bright stars).

Summarizing, we conclude that the overall reliability of the catalog is very high $(>97 \%)$ and that the contamination by spurious sources is strictly restricted to the surrounding areas of very bright sources. For the sake of completeness, we do not remove the sources with $N$ (band) $\leqslant 4$ from the sample. Instead, we include in the data catalog (Table 6) both the number of bands in which the source was detected ( $N$ (band)) and a flag indicating if the source is located in the vicinity of a bright object (see Section 6.2).

\subsection{Star-Galaxy Separation}

Following PG08, we used eight different photometric and morphological criteria to identify stars in the merged photometric catalog: (1) the average of SExtractor star/galaxy separation parameter (stellarity) for all bands where the source is detected; (2) when available, the stellarity parameter and FWHM of the source in the HST images are also considered as an independent, more reliable, criterion; $(3,4)$ the IRAC-based color-magnitude criteria of Eisenhardt et al. (2004) and Barmby et al. (2004); (5) the concentration parameter (i.e., the difference of the [3.6] mag measured in a $3^{\prime \prime}$ radius aperture and the SExtractor MagAuto); (6) the color-color and (7) color-magnitude criteria of Eisenhardt et al. (2004; this time based on optical and NIR bands); and (8) the $B z K$ criteria of Daddi et al. (2004). In spite of using multiple stellarity criteria, the heterogeneous band coverage makes difficult the simultaneous application of the eight criteria (e.g., the HST data cover only $\sim 40 \%$ of the full area). For this reason, we chose the bulk of the criteria to be based on IRAC or optical colors, which are available for the majority of the sources independently of the region in which they are located. We verify that at least five criteria can be estimated for $86 \%$ of the sources with $[3.6]<23.75$.
A source was identified as a star if three or more of the stellarity criteria are satisfied. Based on this method, we found 2913 stars. Among them, we are able to identify $69 \%$ of the spectroscopically confirmed stars. The rest of them satisfy at least one or two of the stellarity criteria. Figure 8 illustrates the efficiency of the IRAC morphological criteria by Eisenhardt et al. (2004) and the $B z K$ criteria of Daddi et al. (2004). Red dots indicate sources with $N$ (criteria) $\geqslant 3$. Clearly, the $B z K$ criterion is more efficient identifying stars over a wider magnitude range. Note that, as discussed in PG08, an IRAC-selected catalog down to [3.6] $<23.75$ only includes a minor fraction of stars (less than $5 \%$ at all magnitudes), the majority of them at bright magnitudes $(\sim 40 \%$ at $[3.6]<18)$. At the faintest magnitudes, the overall dimming of the objects could lead us to identify some stars as galaxies due to the lack of applicable criteria. However, we find that both the galactic and stellar number counts in the IRAC bands are in good agreement with those presented in Fazio et al. (2004) down to our limiting magnitude. From the relative distribution of galaxies and stars, we also find that the fraction of stars makes up for only $3 \%$ of all sources at magnitudes fainter than the median of the sample $([3.6]=22.4)$.

\section{DATA CATALOGS AND DATABASE ACCESS}

The catalog with the multi-band photometry for all the IRAC $3.6+4.5 \mu \mathrm{m}$ selected sample in the EGS is presented in Table 5. Additional information regarding the photometry of the sources and other properties described in the paper are given in Table 6. Furthermore, we also present here a Webbased interface to access our database containing all the results presented in this and the companion paper. The Web tool is publicly available for the entire astronomical community.

As explained in Section 3.2, the catalogs presented in this paper are restricted to the 76,936 sources with [3.6] $<23.75$, the $\sim 85 \%$ completeness level, which count with accurate IRAC photometry $(\mathrm{S} / \mathrm{N}>8)$. As explained in Section 3, our sample is extracted from the area of the IRAC mosaic counting with a frame coverage larger than $20\left(t_{\exp } \sim 4 \mathrm{ks}\right.$ in the IRAC $3.6 \mu \mathrm{m}$ band). Nevertheless, a deeper, although less complete, catalog without any magnitude restriction can be accessed through the online database (Section 6.3). In the following subsections, we describe the contents of Tables 5 and 6 and present Rainbow Navigator.

\subsection{Table 5: Photometric Catalog}

These are the fields included in Table 5.

1. Object. Unique object identifier starting with irac000001. Objects labeled with an underscore plus a number (e.g., irac000356_1) are those identified as a single source in the IRAC catalog built with SExtractor, but deblended during the photometric measurement carried out with the Rainbow software (see Section 4.1). Note that, although the catalog contains 76,936 elements, the identifiers do not follow the sequence irac000001 to irac076185. This is because the catalog is extracted from a larger reference set by imposing coordinate and magnitude constraints. The table is sorted according to this unique identifier.

2. $\alpha, \delta . \mathrm{J} 2000.0$ right ascension and declination in degrees.

3. zspec. Spectroscopic redshift determination drawn from the DEEP2 spectroscopic survey or the catalog of LBGs of Steidel et al. (2003). 

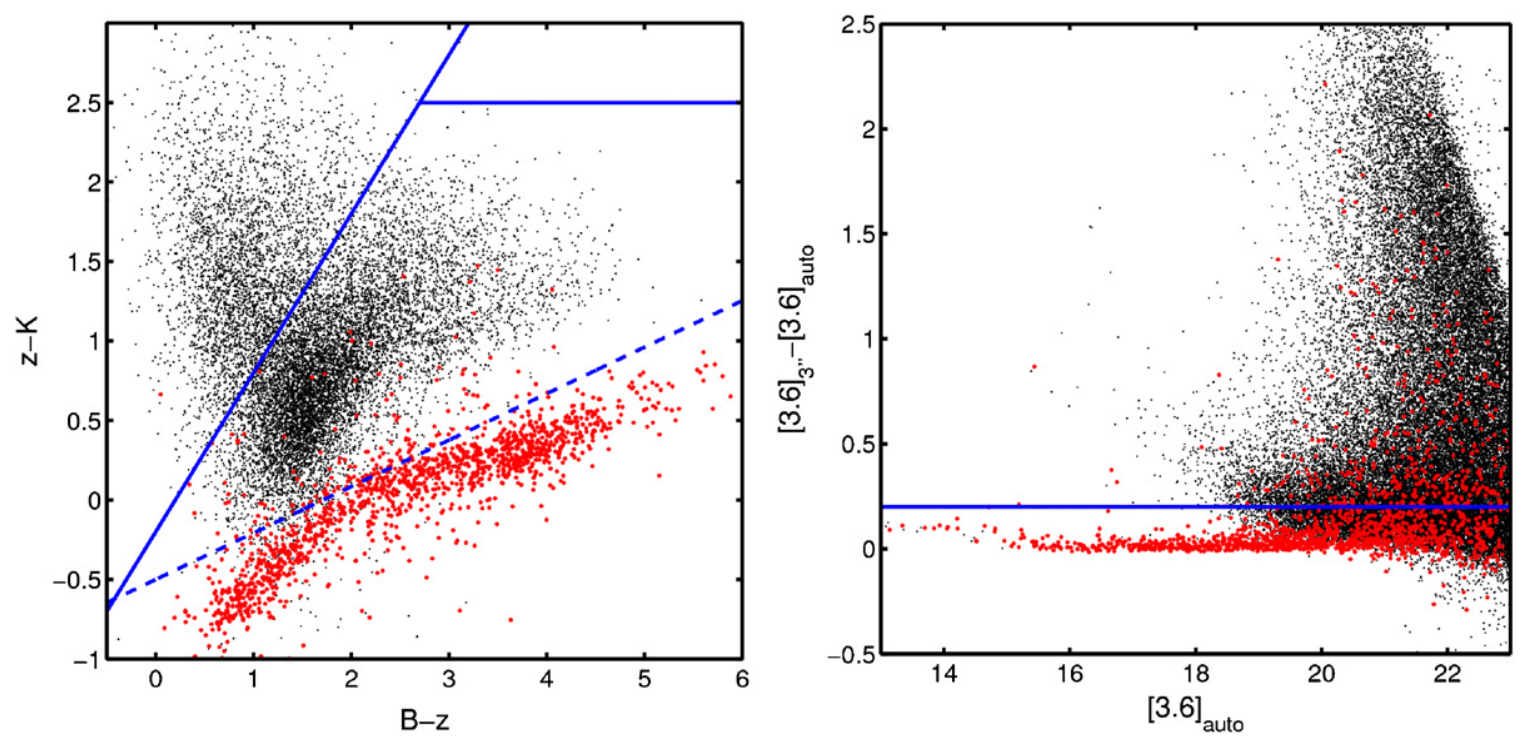

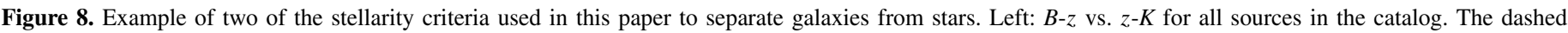

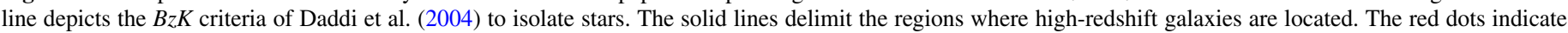

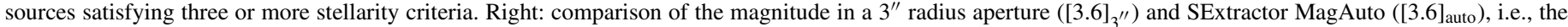

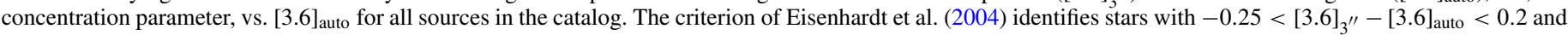
$[3.6]_{\text {auto }}<17.80$.

4. qflag. Spectroscopic redshift quality flag from 1 to 4 . Sources with qflag $>3$ have a redshift reliability larger than $80 \%$.

5. FUV, $N U V, u^{\prime}, \ldots$. Effective wavelengths (in nanometers), magnitudes, and uncertainties (in the $\mathrm{AB}$ system) for each of the 30 photometric bands compiled for this paper. The bandpasses are GALEX FUV and NUV, CFHTLS $u^{*} g^{\prime} r^{\prime} i^{\prime} z^{\prime}$, MMT $u^{\prime} g i z$, CFHT12k BRI, ACS $V_{606}$ and $i_{814}$, Subaru $R$, NICMOS $J_{110}, H_{160}$, MOIRCS $K_{s}$, WIRC $J K$, CAHA- $J K_{s}$ IRAC 3.6-8.0, and MIPS $24 \mu \mathrm{m}$ and $70 \mu \mathrm{m}$. The bands are sorted according to the effective wavelength of the filter. We refer to Section 3 for details on the photometric measurement and error calculation. The magnitudes do not include zero-point corrections (see Paper II, Section 3.1.3). A value of the magnitude equal to -99.0 with error equal to 0.0 indicates a not valid photometric measurement. A negative error indicates that the source was undetected by SExtractor but a positive flux was obtained when forcing the measurement at that position using the appropriate aperture (see Section 4.2). An error equal to 0.0 indicates that the source was undetected and the forced measurement returned a negative flux. In this case, the value of the magnitude is an upper limit equal to the sky $r m s(1 \sigma)$ in the photometric aperture (see Section 4.2).

\subsection{Table 6: Photometric Properties Catalog}

These are the fields included in Table 6.

1. Object. Unique object identifier (the same as in the photometric catalog in Table 5).

2. $\alpha, \delta$. $\mathbf{J} 2000.0$ right ascension and declination in degrees.

3. N(bands,detect). Number of UV-to-NIR in which the source is detected.

4. N(bands,forced). Number of UV-to-NIR in which the source is a priori undetected, but the forced photometry is able to recover a valid flux.

5. Flag. Quality flag indicating that the source is located in the vicinity of a bright object. Sources detected only in the
IRAC bands $(N($ band $)<5)$ and close to a bright $([3.6]>16)$ source are likely to be spurious (see Section 5.3). The values of the flag indicate: (5) source within $70^{\prime \prime}-100^{\prime \prime}$ of the brightness saturated stars in the field, (4) source within $30^{\prime \prime}$ of a [3.6] $<15$ source, (3) source within $20^{\prime \prime}$ of a $15<$ [3.6] $<16$ source, (2) source within $15^{\prime \prime}$ of a $16<[3.6]<17$ source, (1) source within $10^{\prime \prime}$ of a $17<[3.6]<18$ source, and (0) source unflagged.

6. Stellarity. Total number of stellarity criteria satisfied (see Section 5.4). A source is classified as a star if it satisfies three or more criteria. (Section 5.4).

7. Region. Region of the field in which the source is located (Section 5): A value of 1 or 0 indicates that the source is in the main or flanking region, respectively. The main region is defined as the area of the IRAC mosaic within $52.16<\delta<53.20$ and $\alpha>214.04$, the flanking regions are those containing the remaining area.

\subsection{Rainbow Database and Navigator}

The photometric catalog presented here and the inferred stellar parameters discussed in Paper II are obtained using the Rainbow software (see PG05; PG08). The program includes different sub-routines for each task, and the output of each step serves as the input for the next. After the data processing, both the input (images, spectra, templates) and the resulting catalogs are stored in a database with individual sources as building blocks. On doing so, we achieve several goals: (1) each object is fully characterized with all the available information; (2) the data can be easily sorted and retrieved according to several criteria; (3) the data are homogeneously stored, which allows a straightforward combination with data from other Rainbow fields and projects (such as those in PG05 or PG08).

In order to provide worldwide access to the data stored in our database, we have developed a publicly available Web interface, dubbed Rainbow Navigator. ${ }^{12}$ Here we briefly describe the main

\footnotetext{
12 http://rainbowx.fis.ucm.es
} 
The IRAC 3.6+4.5 $\mu \mathrm{m}$ Sample: Multi-band Photometry

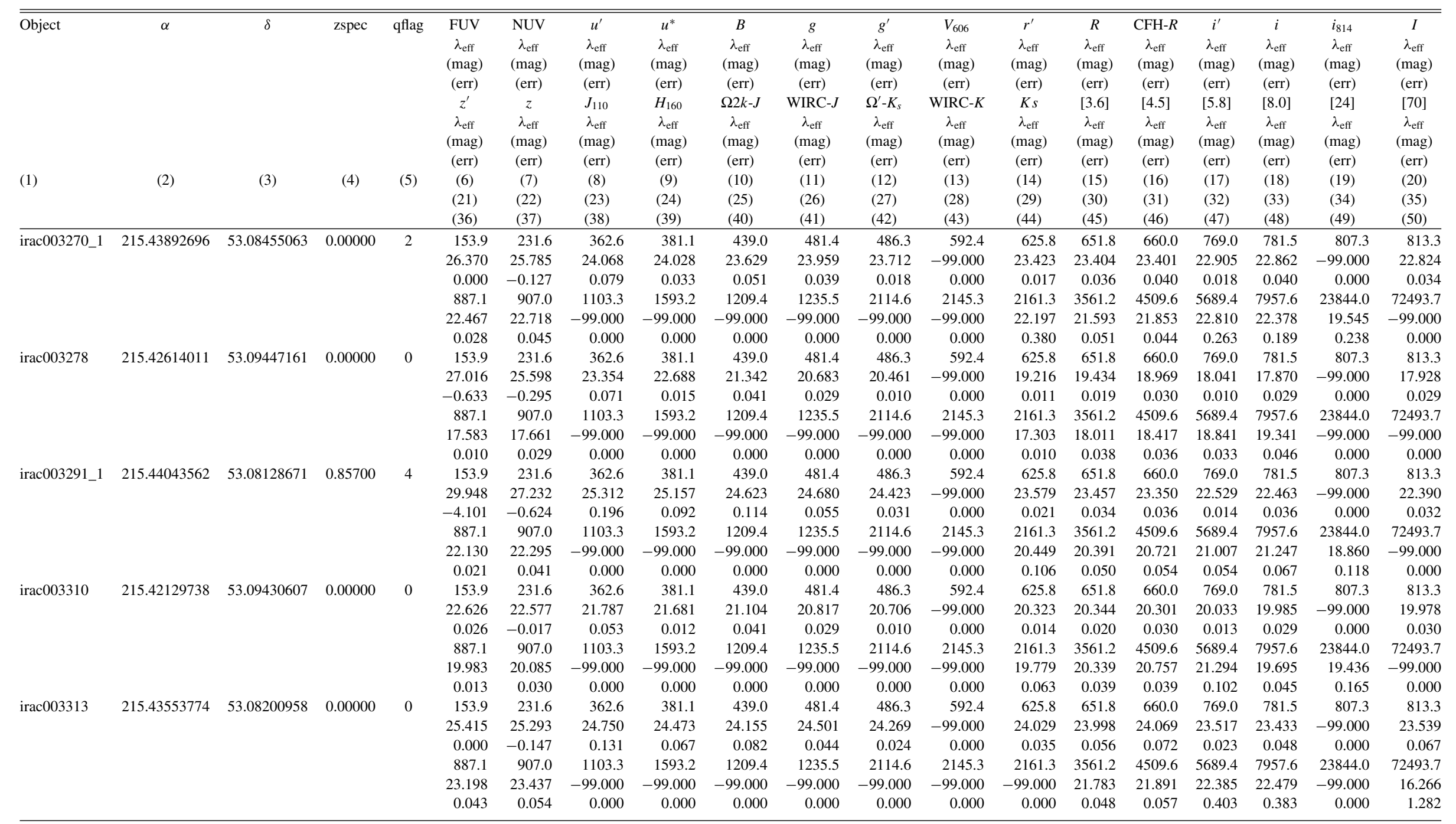

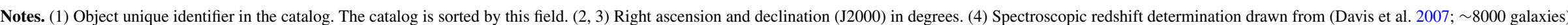

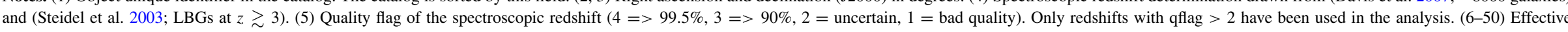

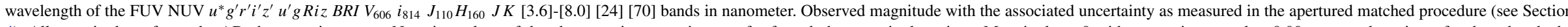

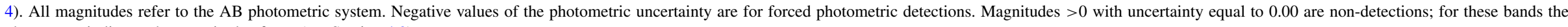
photometry indicates the magnitude of $\sigma_{\text {sky }}$ (see Section 4.2).

(This table is available in its entirety in a machine-readable form in the online journal. A portion is shown here for guidance regarding its form and content.) 
Table 6

The IRAC 3.6+4.5 $\mu \mathrm{m}$ Sample: Photometric Properties

\begin{tabular}{|c|c|c|c|c|c|c|c|}
\hline $\begin{array}{l}\text { Object } \\
\text { (1) }\end{array}$ & $\begin{array}{c}\alpha \\
(2)\end{array}$ & $\begin{array}{c}\delta \\
(3)\end{array}$ & $\begin{array}{c}N(\text { bands, detect) } \\
\text { (4) }\end{array}$ & $\begin{array}{c}N \text { (bands,forced) } \\
(5)\end{array}$ & $\begin{array}{c}\text { Flag } \\
(6)\end{array}$ & $\begin{array}{c}\text { Stellarity } \\
(7)\end{array}$ & $\begin{array}{c}\text { Region } \\
\text { (8) }\end{array}$ \\
\hline irac003270_1 & 215.43910540 & 53.08468920 & 18 & 1 & 0 & 0 & 1 \\
\hline irac003278 & 215.42614011 & 53.09447161 & 18 & 2 & 0 & 7 & 1 \\
\hline irac003291_1 & 215.44058360 & 53.08123980 & 18 & 2 & 0 & 0 & 1 \\
\hline irac003310 & 215.42129738 & 53.09430607 & 19 & 1 & 1 & 0 & 1 \\
\hline irac003313 & 215.43553774 & 53.08200958 & 17 & 1 & 0 & 1 & 1 \\
\hline
\end{tabular}

Notes. (1) Object unique identifier in the catalog. The catalog is sorted by this field. $(2,3)$ Right ascension and declination (J2000) in degrees. (4) Number of optical-to-NIR bands (with effective wavelengths below $8.0 \mu \mathrm{m}$ ) in which the object is detected. (5) Number of optical-to-NIR bands (with effective wavelengths below $8.0 \mu \mathrm{m}$ ) in which the object is a priori undetected, but the forced photometry (see Section 4.2) recovers a valid flux. (6) Quality flag indicating the proximity of a very bright source in the vicinity of the source. Sources detected in very few bands $(N$ (band) $<5$ ) and located nearby a bright source are likely to be spurious detections (see Section 3.2). (7) Sum of all the stellarity criteria satisfied (see Section 5.4). A source is classified as star for Stellarity $>2$. (8) Region of the field in which the source is located: 1 for the main region, 0 for the flanking region.

(This table is available in its entirety in a machine-readable form in the online journal. A portion is shown here for guidance regarding its form and content.)

features of the utility. A detailed description of its capabilities can be found at the Web site.

Rainbow Navigator is essentially a user-friendly Web interface to a database containing all the data products resulting from the process of creating and analyzing the IRAC-selected catalog presented in these two papers, from the initial source detection to the estimate of the stellar parameters.

As many other astronomical query interfaces (e.g., NED, SIMBAD), Rainbow Navigator allows to retrieve the information for single sources searching for the source name or by coordinates. It also allows to create subsets of the complete catalog based on multiple constraints over the multi-band photometry, the redshifts or the stellar parameters. In addition, we have incorporated a cross-matching tool that allows to compare catalogs uploaded by the user to the IRAC-selected sample stored in the database, returning the sources in common. Rainbow Navigator also has an on-the-fly utility to create sky maps of a selected area, including point-and-click access to the individual sources.

Furthermore, an interesting feature that we do not include in the public catalog for the sake of simplicity is the possibility of retrieving observed and rest-frame synthetic magnitudes over a predefined grid of 52 different filters covering the whole spectral range from the UV to the radio wavelengths. These values are computed by convolving the best-fitting template (see Paper II) for each source with the appropriate filter transmission curve.

Each source of the catalog has its own data sheets that provide all the available information including not only the photometry, the redshifts, or the stellar parameters but also detailed information of the stellarity, synthetic magnitudes, and the multiplicity in other bands, jointly with the unique identification and coordinates of the counterparts in each given band. The tool also provides postage stamps of the source in each of the available bands that can be modified on-the-fly or combined to create simple false color images. Furthermore, each page includes a figure depicting the full SED showing the fit of the optical and IR data jointly with the best-fitting templates.

Figures 9-12 show examples of the multi-band postage stamps, the UV-to-FIR SED and fitting templates, and the clickable map utility for a few galaxies at different redshifts. The postage stamps also illustrate the different photometric measurements in each band (aperture matched and circular apertures) and the forced detections for very faint sources (see, e.g., the source in Figure 11).
Rainbow Navigator has been conceived to serve as a permanent repository for future versions of the catalogs containing improvements over the previous results (the present version is data release 1), and also to similar data products in other cosmological fields (such as GOODS-N and GOODS-S, presented in PG08). Currently, it provides public access to the IRAC-selected catalog in the EGS presented in this paper, and also a similar release of the data described in PG08 for a small piece of the central region of the GOODS-S region.

\section{SUMMARY}

We presented an IRAC $3.6+4.5 \mu \mathrm{m}$ selected catalog in the EGS characterized with multi-wavelength photometry. The sample contains 76,936 sources with [3.6] $<23.75(85 \%$ completeness of the sample) covering an area of $0.48 \mathrm{deg}^{2}$. The IRAC sources are characterized with FUV NUV $u^{*} g^{\prime} r^{\prime} i^{\prime} z^{\prime}$ $u^{\prime} g R i z$ BRI $V_{606} i_{814} J_{110} H_{160} J K$ [3.6]-[8.0] photometry. In addition, we have cross-correlated the sample with X-ray data (Laird et al. 2009, AEGIS-X), Spitzer/MIPS $24 \mu \mathrm{m}$ and $70 \mu \mathrm{m}$ FIR photometry, and VLA $20 \mathrm{~cm}$ radio data (AEGIS20; Ivison et al. 2007). Secure spectroscopic redshifts are also included in the catalog for 7636 sources with [3.6] $<23.75$ obtained from the DEEP2 Survey and Steidel et al. (2003; LBGs at $z \gtrsim 3$ ). The data described in this paper are publicly available, and will be part of future extended analysis and projects. The main results of this work are summarized below.

1. The extraction of the IRAC sample presented in this paper was limited to a region with exposure times $>4 \mathrm{ks}$. The average survey depth is $t_{\exp } \sim 10 \mathrm{ks}$. Aperture photometry was performed in the four IRAC bands simultaneously allowing us to obtain upper limit fluxes for undetected sources in the [5.8] and [8.0] bands. We removed spurious detections masking areas around bright stars. The estimated $85 \%$ completeness level for point sources is [3.6,4.5] $\sim 23.75$ and $[4.5,5.8] \sim 22.25$. The $3 \sigma$ limiting magnitude estimated from the sky rms is $[3.6,4.5] \sim 24.75$ and $[4.5,5.8] \sim 22.90$. We also validated the quality of the photometry by comparing our results with the catalog of Barmby et al. (2008), finding good agreement in both magnitudes $(\lesssim 0.05 \mathrm{mag}$ ) and uncertainties (Section 3.3). Some small systematics were found in this comparison, which can be attributed to the slightly different 

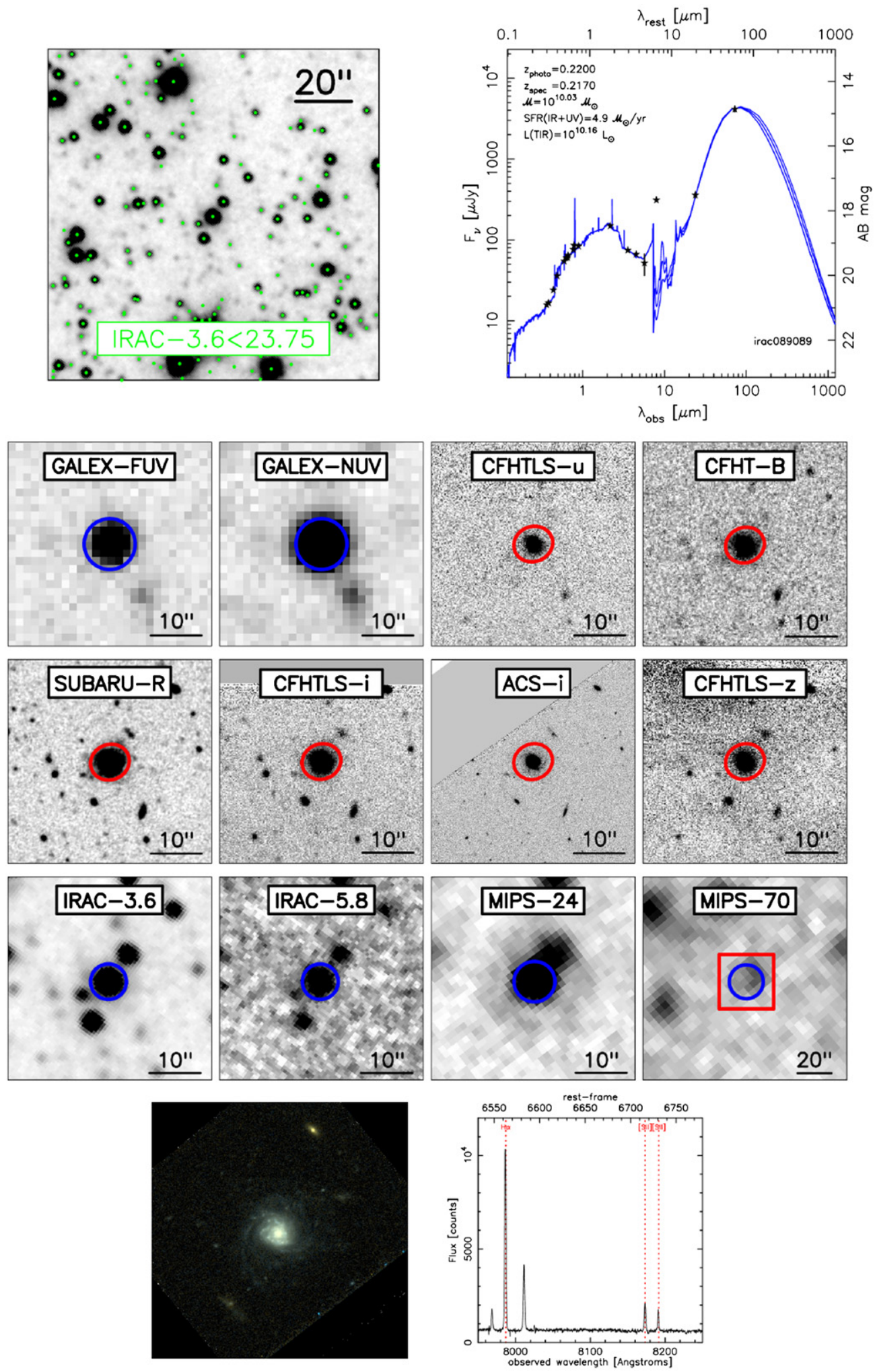

Figure 9. Example of the results available for each IRAC source in the Rainbow database, accessible through the Rainbow Navigator interface. This source is irac089089, a galaxy at $z=0.2170$. Top left: map $\left(2^{\prime} \times 2^{\prime}\right)$ of the sky area around the source. The green dots in the map depict sources in our IRAC catalog with [3.6] < 23.75. By clicking on any of the sources, the interface presents an individual Web site with all the available information for that source. Top right: full UV-to-FIR SED of the central galaxy in the sky map, a source at $z=0.21$. The UV-to-NIR data are fitted to a stellar population model, while the IR-part of the SED is fitted to the models of CE01, DH02. A summary of the estimated stellar parameters, such us the stellar mass or the global SFR, is shown in the upper-left corner of the figure. Middle panels: gray-scale postage stamps (with size $40^{\prime \prime} \times 40^{\prime \prime}$, except for the MIPS 70 image, whose size is $2^{\prime} \times 2^{\prime}$, same as the map in the top left figure) of the galaxy in some of the available bands, covering different wavelength ranges. The Kron aperture used to measure consistent photometry in optical/NIR bands and the circular aperture used in bands with significantly lower resolution are shown in all panels (red and blue, respectively). Bottom left: RGB color stamp obtained by combining images in the ACS- $V_{606}, i_{814}$ bands. The Rainbow Navigator Web interface allows to produce on-the-fly monochromatic and RGB images changing the cuts interactively. Bottom right: DEEP2 1-D spectra of the galaxy depicting some of the identified lines. The wavelength range and the redshift can be modified interactively. 

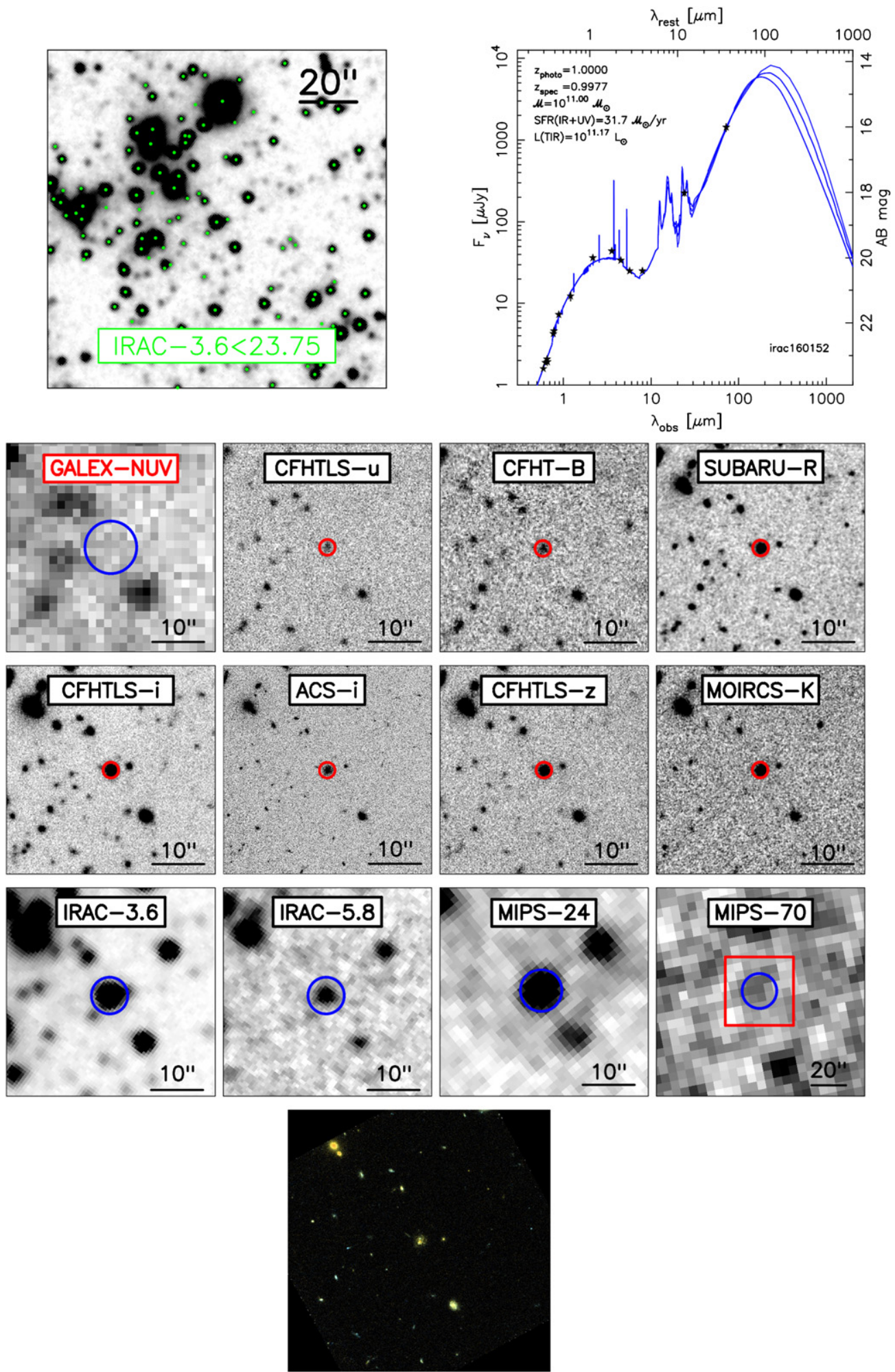

Figure 10. Same as Figure 9 for the galaxy irac160152 at $z=0.99$. The band label shown in red in the GALEX-NUV postage stamps indicates a non-detection. For each of these bands, $\sigma_{\text {sky }}$ is depicted in the UV-to-FIR SED as red arrow. Green name labels indicate forced detections (see Section 4.2). These bands are shown as green stars in the SED plot.

reduction versions and the limiting depth of the source extractions.

2. We described in detail our custom photometric procedure Rainbow, developed to measure photometry in multiwavelength data in a consistent way. The main steps followed by our photometry software are: (a) re-calibration of the local $\left(5^{\prime} \times 5^{\prime}\right)$ astrometric solution for each pair of images, improving the accuracy of the cross-identification of sources to a limit below $0^{\prime \prime} 1(0,2)$ between opticalNIR (IRAC-ground based) images. (b) Deconvolution of blended IRAC sources $(\sim 16 \%$ of the entire catalog) separated by more than $1^{\prime \prime}$, using the positions of the 

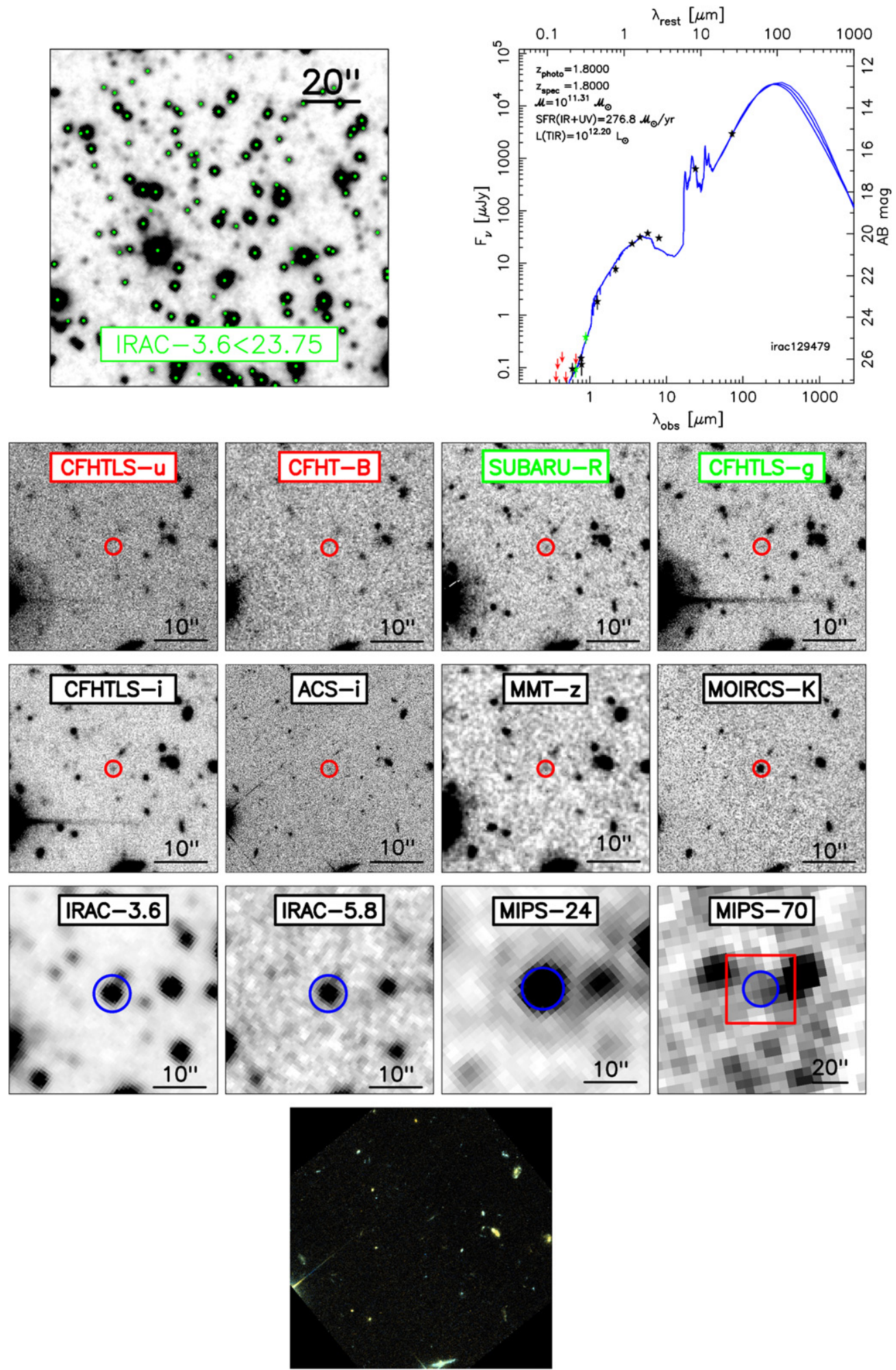

Figure 11. Same as Figure 9 for irac129479, an IR-bright galaxy at $z=1.8$. This galaxy corresponds to source EGS11 in the paper of Huang et al. (2009). Band labels in red in the gray-scale postage stamps indicate non-detections. For each of these bands, $\sigma_{\text {sky }}$ is depicted in the UV-to-FIR SED as red arrow. Band labels in green indicate forced photometric measurements (see Section 4.2). These bands are shown as green stars in the SED plot.

optical/NIR counterparts as reference. The IRAC photometry of deblended sources is accurate to $0.03-0.10 \mathrm{mag}$, depending on the flux ratio between neighbors (Section 4.1). (c) Measurement of consistent aperture matched photometry for a wide range of ground- and spacebased observations, with different depths and resolutions (Section 4.2). (d) Obtaining (forced) flux measurements and upper limits for faint, undetected (in a direct analysis of each image) sources (Section 4.2). (e) Computing robust photometric errors that take into account variations in the sky rms and signal correlation (Section 4.3).

3. The inhomogeneous multi-wavelength coverage of the region covered by IRAC and the differences in depth of the various data sets justify the split of the complete sample 

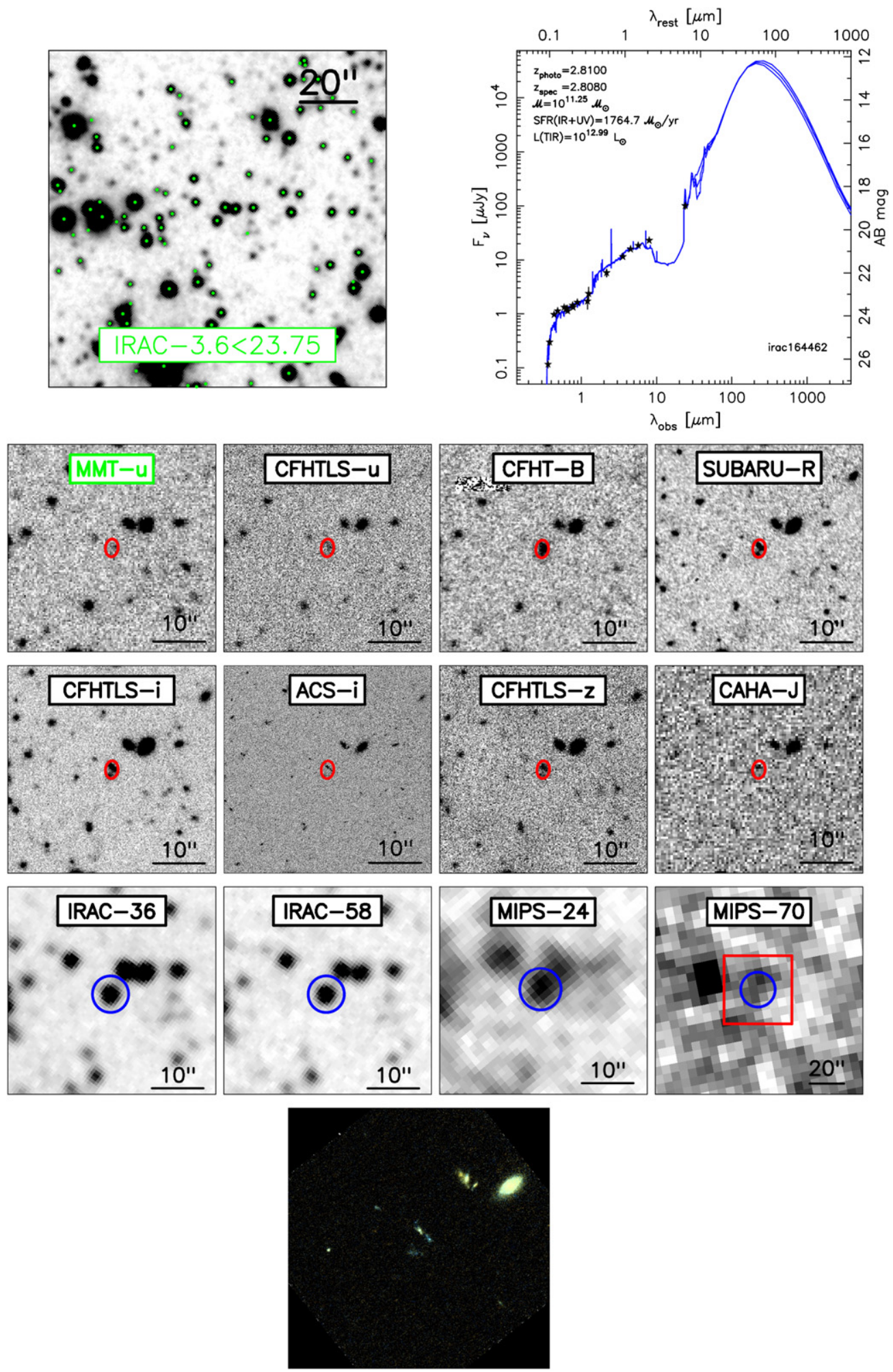

Figure 12. Same as Figure 11 for irac164462, an LBG at $z=2.8$. Band labels in red in the gray-scale postage stamps indicate non-detections. For each of these bands, $\sigma_{\text {sky }}$ is depicted in the UV-to-FIR SED as red arrow. Band labels in green indicate forced photometric measurements (see Section 4.2). These bands are shown as green stars in the SED plot.

in two complementary regions. The main region (covering $0.35 \mathrm{deg}^{2}$ ), delimited by the footprint of the CFHTLS image, constitutes the region with the highest data quality. Here the SEDs present a median coverage of 19 bands, including high-resolution imaging from $H S T$ (for $\sim 50 \%$ of the region) and deep NIR data from Subaru-MOIRCS (for $\sim 25 \%$ of the region). Nearly $\sim 70 \%$ of the complete sample presented in this paper is located in the main region. In the flanking regions $\left(0.13 \mathrm{deg}^{2}\right)$, the optical-to-NIR coverage is still robust, but the median-band coverage is reduced to 11 bands, lacking the high-resolution $H S T$ data and the deepest NIR observations. 
4. The overall detection efficiency of counterparts for the IRAC sources $([3.6]<23.75)$ in other bands is high: more than $85 \%$ of the sources are detected (with $\mathrm{S} / \mathrm{N}>5$ ) in the deepest optical data ( $R$ or $i^{\prime}$ bands) and $70 \%$ in the deepest (MOIRCS) $K_{s}$-band images. Our method to perform (forced) photometric measurements for a priori undetected sources allows us to recover $10 \%-20 \%$ additional sources in the shallowest images. Despite the large fraction of IRAC sources detected in all other bands, we find that $\sim 2 \%$ of the sample is detected in IRAC only, some of them at relatively bright magnitudes $21<$ [3.6] $<22$ and with high $\mathrm{S} / \mathrm{N}$.

5. Around $10 \%$ of the sample counts with reliable spectroscopic redshifts. Nearly $20 \%$ and $2 \%$ of the sources are detected in the MIPS $24 \mu \mathrm{m}$ and MIPS $70 \mu \mathrm{m}$ data, respectively. This allows a detailed analysis of their IR-based SFRs. In addition, we recover 1023 of the X-ray sources in the catalog published by Laird et al. (2009; $77 \%$ of their complete catalog) and 590 radio sources from the catalog published by Ivison et al. (2007; 52\% of their entire sample).

6. We presented the publicly available Web interface, Rainbow Navigator, for the database containing all the data products from this and the forthcoming companion paper (Paper II). The interface allows the access to the data using customizable queries on the photometry and derived parameters. Furthermore, it provides additional capabilities to inspect the data, such as the creation of on-the-fly clickable sky maps or the cross-match of the IRAC sample presented here to a user-provided catalog. We have made a significant effort to develop a useful and accessible tool that maximizes the legacy value of our catalogs in the EGS, and the future Rainbow based projects in other relevant fields (e.g., PG08). The online version of the catalog (IRAC-DR1) contains a deeper, although less complete, sample limited to $[3.6]<25$.

7. In Paper II, we make use of the very detailed UV-to-FIR SEDs presented here to estimate photometric redshifts, stellar masses, and SFRs.

We acknowledge support from the Spanish Programa Nacional de Astronomía y Astrofísica under grant AYA200910368. P.G.P.-G. acknowledges support from the Ramón y Cajal Program financed by the Spanish Government and the European Union. Partially funded by the Spanish MICINN under the Consolider-Ingenio 2010 Program grant CSD2006-00070: First Science with the GTC. Support was also provided by NASA through contract no. 1255094 issued by JPL/Caltech. This work is based in part on observations made with the Spitzer Space Telescope, which is operated by the Jet Propulsion Laboratory, Caltech under NASA contract 1407. Observations reported here were obtained at the MMT Observatory, a joint facility of the Smithsonian Institution and the University of Arizona. GALEX is a NASA Small Explorer launched in 2003 April. We gratefully acknowledge NASA's support for construction, operation, and scientific analysis of the GALEX mission. This research has made use of the NASA/IPAC Extragalactic Database (NED) which is operated by the Jet Propulsion Laboratory, California Institute of Technology, under contract with the National Aeronautics and Space Administration. Based in part on data collected at Subaru Telescope and obtained from the SMOKA, which is operated by the Astronomy Data Center, National Astronomical Observatory of Japan.

\section{REFERENCES}

Barmby, P., et al. 2004, ApJS, 154, 97

Barmby, P., et al. 2008, ApJS, 177, 431

Barro, G., et al. 2009, A\&A, 494, 63

Bertin, E., \& Arnouts, S. 1996, A\&AS, 117, 393

Bundy, K., et al. 2006, ApJ, 651, 120

Capak, P., et al. 2004, AJ, 127, 180

Caputi, K. I., et al. 2007, ApJ, 660, 97

Chary, R., \& Elbaz, D. 2001, ApJ, 556, 562

Coil, A. L., et al. 2004, ApJ, 617, 765

Colina, L., \& Bohlin, R. C. 1994, AJ, 108, 1931

Cristóbal-Hornillos, D., et al. 2003, ApJ, 595, 71

Daddi, E., et al. 2004, ApJ, 617, 746

Daddi, E., et al. 2007, ApJ, 670, 156

Davis, M., et al. 2003, Proc. SPIE, 4834, 161

Davis, M., et al. 2007, ApJ, 660, L1

Eisenhardt, P. R., et al. 2004, ApJS, 154, 48

Faber, S. M., et al. 2003, Proc. SPIE, 4841, 1657

Fazio, G. G., et al. 2004, ApJS, 154, 39

Gawiser, E., et al. 2006, ApJS, 162, 1

Giavalisco, M., et al. 2004, ApJ, 600, L93

Grazian, A., et al. 2006, A\&A, 449, 951

Gwyn, S., et al. 2011, arXiv:1101.1084

Huang, J., et al. 2004, ApJS, 154, 44

Huang, J., et al. 2009, ApJ, 700, 183

Iglesias-Páramo, J., et al. 2007, ApJ, 670, 279

Ilbert, O., et al. 2006, A\&A, 457, 841

Ilbert, O., et al. 2009, ApJ, 690, 1236

Ivison, R. J., et al. 2007, ApJ, 660, L77

Kajisawa, M., et al. 2009, ApJ, 702, 1393

Kron, R. G. 1980, ApJS, 43, 305

Labbé, I., et al. 2003, AJ, 125, 1107

Laird, E. S., et al. 2009, ApJS, 180, 102

Le Fèvre, O., et al. 2005, A\&A, 439, 845

Lotz, J. M., et al. 2008, ApJ, 672, L177

Mancini, C., et al. 2009, A\&A, 500, 705

Martin, D. C., et al. 2005, ApJ, 619, L1

McLeod, B., et al. 2006, in Scientific Detectors for Astronomy 2005, ed. J. E. Beletic, J. W. Beletic, \& P. Amico (Berlin: Springer), 337

Miyazaki, S., et al. 2007, ApJ, 669, 714

Muzzin, A., et al. 2009, ApJ, 701, 1839

Nandra, K., et al. 2005, MNRAS, 356, 568

Oke, J. B., et al. 1995, PASP, 107, 375

Pérez-González, P. G., et al. 2005, ApJ, 630, 82

Pérez-González, P. G., et al. 2008, ApJ, 675, 234

Pozzetti, L., et al. 2007, A\&A, 474, 443

Quadri, R., et al. 2007, AJ, 134, 1103

Reach, W. T., et al. 2005, PASP, 117, 978

Reddy, N. A., Steidel, C. C., Erb, D. K., Shapley, A. E., \& Pettini, M. 2006, ApJ, 653, 1004

Rieke, G. H., et al. 2004, ApJS, 154, 25

Rodighiero, G., et al. 2007, A\&A, 470, 21

Rowan-Robinson, M., et al. 2008, MNRAS, 386, 697

Salim, S., et al. 2007, ApJS, 173, 267

Santini, P., et al. 2009, A\&A, 504, 751

Scoville, N., et al. 2007, ApJS, 172, 1

Steidel, C. C., Giavalisco, M., Pettini, M., Dickinson, M., \& Adelberger, K. L. 1996, ApJ, 462, L17

Steidel, C. C., et al. 2003, ApJ, 592, 728

Villar, V., et al. 2008, ApJ, 677, 169

Walcher, C. J., et al. 2008, A\&A, 491, 713

Wang, W., Cowie, L. L., Barger, A. J., Keenan, R. C., \& Ting, H. 2010, ApJS, 187,251

Williams, R. J., Quadri, R. F., Franx, M., van Dokkum, P., \& Labbé, I. 2009, ApJ, 691, 1879

Williams, R. E., et al. 1996, AJ, 112, 1335

Wolf, C., et al. 2001, A\&A, 377, 442

Wuyts, S., et al. 2007, ApJ, 655, 51

Wuyts, S., et al. 2008, ApJ, 682, 985

Yan, H., et al. 2004, ApJ, 616, 63

York, D. G., et al. 2000, AJ, 120, 1579

Zhao, Y., Huang, J., Ashby, M., Fazio, G., \& Miyazaki, S. 2009, Res. Astron. Astrophys., 9, 1061 